\title{
Cosmological-scale coherent orientations of quasar optical polarization vectors in the Planck era Investigating the Galactic dust contamination scenario
}

\begin{abstract}
V. Pelgrims
Univ. Grenoble Alpes, CNRS, Grenoble INP, LPSC-IN2P3, 38000 Grenoble, France

e-mail: pelgrims@lpsc.in2p3.fr

Received 2 June 2017 / Accepted 30 November 2018

ABSTRACT

Gigaparsec scale alignments of the quasar optical polarization vectors have been proven to be robust against a scenario of contamination by the Galactic interstellar medium (ISM). This claim has been established by means of optical polarization measurements of the starlight surrounding the lines of sight of the 355 quasars for which reliable optical polarization measurements are available. In this paper, we take advantage of the full-sky and high quality polarization data released by the Planck satellite to provide an independent, complementary, and up-to-date estimation of the contamination level of the quasar optical polarization data by the Galactic dust. Our analysis reveals signatures of Galactic dust contamination at the two sigma level for about 30 percent of the quasar optical polarization data sample. The remaining 70 percent of the lines of sight do not show Galactic dust contamination above the two sigma level, suggesting low to negligible contamination of the quasar optical polarization signal. We further found arguments suggesting that Galactic thermal dust cannot fully account for the reported quasar optical polarization alignments. Based on the measurements of the ratio of the polarized intensity of the dust in the submillimeter to the degree of linear polarization of the quasar in the optical, we provide a new and independent quality criteria to apply to the quasar optical polarization sample. We argue that, unless correction is applied, such a criterion should be imposed on the data for future investigations in the framework of the cosmological-scale correlations of quasar optical polarization vector orientations that still could compete with the isotropic principle of the cosmological paradigm.
\end{abstract}

Key words. polarization - quasars: general - submillimeter: ISM - dust, extinction - large-scale structure of Universe ISM: magnetic fields

\section{Introduction}

Hutsemékers (1998), Hutsemékers \& Lamy (2001) and Hutsemékers et al. (2005) have reported alignments of quasar optical linear polarization vectors extending over cosmological-scale regions of the Universe (see also Jain et al. 2004; Pelgrims \& Cudell 2014; Hutsemékers et al. 2014; Pelgrims \& Hutsemékers 2015, 2016; Pelgrims 2016 for a recent review). The very large scales at which these coherent orientations take place suggest a cosmological origin or at least a correlation between objects or fields over cosmological scales, up to $\sim 1 \mathrm{Gpc}$ at redshift $z \simeq 1-2$. These observations were recognized as reflecting the need to add new ingredients to the well-accepted cosmological concordance model. Since their observational discovery, a wide variety of scenarios have been proposed to explain these alignments. A nonexhaustive list of these includes fundamental constant variations, cosmic strings, cosmic birefringence, cosmological magnetic fields, new dark matter particle candidates, anisotropic or rotational cosmological models, and bad sampling or contamination of the data set (see, e.g., Chang et al. 2012; Poltis \& Stojkovic 2010; di Serego Alighieri 2015; Urban \& Zhitnitsky 2010; Tiwari \& Jain 2015; Das et al. 2005; Payez et al. 2008; Hutsemékers et al. 2011, 2005; Agarwal et al. 2011; Ciarcelluti 2012; Kuvschinova \& Panov 2014; Joshi et al. 2007). It is worth mentioning that none of these scenarios have been able yet to reproduce fully the observational characteristics of these alignments (e.g., Hutsemékers et al. 2010; di Serego Alighieri 2015).
Because the polarization vectors of objects located in the same region of the sky but at different redshift do not show the same alignment direction, it has been argued that possible instrumental bias and contamination by the interstellar medium (ISM) are unlikely to be responsible for the observed polarization angle correlations (Hutsemékers 1998; Hutsemékers \& Lamy 2001; Hutsemékers et al. 2005; Payez et al. 2010). The eventuality of instrumental bias has been discussed by Hutsemékers (1998), Hutsemékers et al. (2005) and Sluse et al. (2005). Briefly, their conclusion is that instrumental bias is very unlikely because the quasar optical polarization sample contains data from different observational campaigns, measured by different authors, using different techniques and/or with different instruments. Additionally, the global significance of the patterns of polarization alignments has been assessed using dedicated statistical techniques, specifically based on the reshuffling of the polarization vectors on the source positions that would have taken a global bias into account when computing the prior of randomness (see Jain et al. 2004; Hutsemékers et al. 2005).

As discussed further in Sect. 3, the combination of magnetic fields and dust grains in our Galaxy is well known to polarize optical light from distant stars and to be at the origin of alignment patterns of star polarization vectors (e.g., Mathewson \& Ford 1970; Axon \& Ellis 1976; Planck Collaboration Int. XXI 2015; Planck Collaboration XII 2018). While the quasar light is certainly contaminated to some degree by the traversed ISM, Hutsemékers (1998) and Hutsemékers et al. (2005) made sure it cannot be at the origin of the large-scale quasar polarization 
vector alignments. First, they used Burstein \& Heiles (1982) extinction maps to evaluate the contamination of the quasar polarization data by the ISM. As a consequence they imposed severe selection criteria on the quasar polarization data to eliminate at best potentially strong contaminated data points. Second, they considered the polarized Galactic star catalogs of Axon \& Ellis (1976) and Heiles (2000) to compare the quasar polarization vector orientations with those of the nearest polarized Galactic stars. The detection of the large-scale alignments of the quasar polarization vectors were found to be robust against the scenario of a Galactic dust contamination. A similar conclusion was also found in Payez et al. (2010) where a basic modeling of the ISM contamination was discussed.

In this paper, our aim is to provide independently and with up-to-date data sets a new estimate of the degree of contamination of the quasar optical polarization sample by the Galactic dust and thus to test further the possibility following which Galactic dust could be at the origin of the reported alignments. For this purpose, we make use of the diffuse thermal dust polarized emission measured by the Planck satellite ${ }^{1}$ at $353 \mathrm{GHz}$ (Planck Collaboration Int. XIX 2015). Compared to previous studies that rely on starlight polarization, the use of the Planck polarized dust data has the considerable advantage of probing the whole line of sight through the Milky Way. Some differences between the results obtained using starlight polarization and thermal dust are thus to be expected. Another advantage of considering the maps of the dust polarized emission is from the angular resolution. We may indeed probe rapid spatial variations (on sky) of dust column density and/or of the grain alignment direction that would have been otherwise missed using sparse starlight polarization data. This analysis should yield independent quality criteria for the quasar data and possibly allows, in the future, for the refinement of our understanding of these striking alignments of the quasar polarization vectors.

The paper is structured as follow. In Sect. 2, we present the data sets that we use to evaluate the ISM contamination of the quasar optical polarization data. Sections 3 and 4 contain the core of our analysis and we present and discuss thereby the main results with caveats. We conclude in Sect. 5 and give perspective for future work. Appendix A contains details about our treatment of the dust polarization maps. The robustness of our analysis regarding the choice of the adopted smoothing length of the polarization maps is discussed in Appendices B and C. In Appendix D, we reproduce the main results presented in the rest of the paper but obtained by means of another polarization ratio discussed by Martin (2007) and used in Planck Collaboration Int. XXI (2015).

\section{Data sets}

\subsection{Quasar optical polarization catalog}

The current sample of quasar optical polarization measurements from which the very large-scale alignments of the polarization vectors are studied is made of 355 sources. This sample contains reliable polarization measurements for quasars located in both Galactic hemispheres: 195 quasars are located in the northern hemisphere and 160 in the southern hemisphere. The quasars that make the sample have been selected to avoid at best the ISM Galactic contamination according to the following requirements: $|b| \geq 30^{\circ}, p_{V} \geq 0.6 \%$ and $\sigma_{\psi_{\mathrm{V}}} \leq 14^{\circ}$, which corresponds to about $p_{V} \geq 2 \sigma_{p_{V}} ; b$ is the Galactic latitude of the source; $p_{V}$

\footnotetext{
1 http://sci.esa.int/planck/
}

the degree of linear polarization; $\sigma_{p_{V}}$ its error; and $\sigma_{\psi_{\mathrm{V}}}$ the error on the polarization position angle. We denote the quasar optical polarization position angle $\psi_{V}$, which is defined in the IAU convention (east of north) and is expressed in degree. It takes values in the range $0-180^{\circ}$. The subscript $V$ is used throughout for quasar optical polarization quantities. This choice also indicates that the quasar data are from the optical domain, mostly in the $V$ band (Sluse et al. 2005).

The redshift of the quasars of the final sample varies from 0.06 to 3.95 . Even though all Galactic longitudes are covered, the sky coverage of the sample is unfortunately not homogeneous (see Hutsemékers et al. 2005; Pelgrims 2016 for details). Up to now and to the best of our knowledge it is the only publicly available ${ }^{2}$ large and reliable sample of linear polarization data of quasars at optical wavelengths. Hutsemékers et al. (2005) provide a detailed description of this sample, which consists in a compilation of "new" observations obtained at that time (Hutsemékers 1998; Hutsemékers \& Lamy 2001; Hutsemékers et al. 2005; Sluse et al. 2005) and of ancillary data from the literature.

To proceed to our analysis, we first convert the quasar sky coordinates from B1950 to the J2000. Then, we convert them into Galactic coordinates $(l, b)$. The quasar polarization position angles are transformed accordingly using, for example, Eq. (16) of Hutsemékers (1998).

\subsection{Planck $353 \mathrm{GHz}$ polarized sky and dust data}

The diffuse thermal dust polarized emission is the dominant Galactic foreground present in measurements of the polarization of the cosmic microwave background (CMB) emission at frequencies above $100 \mathrm{GHz}$ (e.g., Planck Collaboration Int. XXII 2015). The Planck satellite recorded this emission and it provided unprecedented full-sky maps of this Galactic emission in intensity $I_{\mathrm{S}}$ and polarization, measuring the $Q_{\mathrm{S}}$ and $U_{\mathrm{S}}$ Stokes parameters. We use the subscript $S$ to refer to the polarization quantities in the submillimeter.

We use the Planck second release of the single-frequency polarization maps at $353 \mathrm{GHz}$ that are available on the Planck Legacy Archive ${ }^{3}$. Planck Collaboration Int. XIX (2015) and Planck Collaboration Int. XXI (2015) give details and discussions regarding these data. The Planck DR2 HFI $353 \mathrm{GHz}$ maps have a native resolution ${ }^{4}$ of about 4.94' (Planck Collaboration Int. XIX 2015) and a HEALPix ${ }^{5}$ grid tessellation corresponding to $N_{\text {side }}=2048$ (Górski et al. 2005). At the instrument resolution, the $353 \mathrm{GHz}$ polarization maps are noise dominated (Planck Collaboration Int. XIX 2015). This is particularly true at high Galactic latitudes $\left(|b| \geq 30^{\circ}\right)$ where the ISM is more diffuse and has a low density. In order to increase the signal-to-noise ratio $(\mathrm{S} / \mathrm{N})$ of the Planck HFI measurements of the diffuse ISM we smooth the Stokes parameter maps. However, smoothing the Planck data accentuates the difference between the dust beam relative to the quasar pencil beam. From a physical point of view, the larger the smoothing value we adopt, the larger the angular scales of the ISM variations we consider as we dilute any smallscale spatial structures which, however, might be important in our case. Therefore, for our comparative analysis to be meaningful, there is a compromise to be reached between maintaining high

\footnotetext{
2 http://cdsarc.u-strasbg.fr/viz-bin/qcat?J/A+A/441/ 915

3 http://pla.esac.esa.int/pla/\#maps

4 https://wiki.cosmos.esa.int/planckpla2015/index.php/ HFI_\performance_summary

5 See http://healpix. sourceforge.net
} 
resolution data and dealing with reliable dust polarization quantities. This is especially true in our case given that smoothing of the maps could lead to lower polarized intensities due to beam depolarization and, consequently, biases in our results.

As shown in Appendix A, we found that it was convenient to smooth the polarization maps with a Gaussian kernel of full width at half maximum (FWHM) of $15^{\prime}$; owing to the intrinsic resolution of the map, the effective beam is then about $15.8^{\prime}$. In order to check the robustness of our results regarding the adopted smoothing kernel value, we also conducted the analyses presented in Sects. 3 and 4 in parallel using a smoothing kernel with FWHM of $5^{\prime}, 10^{\prime}$, and $20^{\prime}$. Globally, the results with the different kernel FWHM values are very consistent as shown in the appendices.

At $353 \mathrm{GHz}$, thermal dust is, by large, the principal source of the polarized signal. The dispersion arising from CMB polarization anisotropies is much lower than the instrumental noise for $Q_{\mathrm{S}}$ and $U_{\mathrm{S}}$ (Planck Collaboration VI 2014). Its impact on our analysis is thus expected to be negligible. The cosmic infrared background (CIB) is assumed to be unpolarized (Planck Collaboration XXX 2014). At this frequency, only small contributions are expected in the intensity map from the CMB, CIB, and zodiacal light (e.g., Planck Collaboration Int. XXI 2015). As we used only the intensity map to reinforce other results, we found that it was unnecessary to correct the map from these contributions.

For each quasar we identified the HEALPix pixel corresponding to its position on the sky and we estimated the thermal dust emission quantities from the smoothed maps. We note that quasars of our sample are point-like sources having angular size below the arcsecond level (Sluse et al. 2005). Their contributions, or the contributions of their host Galaxy, to the $353 \mathrm{GHz}$ signal collected by Planck are considered as negligible because of the instrument resolution at $353 \mathrm{GHz}$, which is the scale at which diffuse Galactic components dominate largely the signal. Their contributions are indeed diluted by the Planck beam, a situation that is enhanced by the additional smoothing of the data that we performed.

From the $Q_{\mathrm{S}}$ and $U_{\mathrm{S}}$ of the smoothed maps, we derived the dust (linear) polarized intensity $P_{\mathrm{S}}$, computed as $P_{\mathrm{S}}^{2}=Q_{\mathrm{S}}^{2}+U_{\mathrm{S}}^{2}$, and $\psi_{\mathrm{S}}$ the dust polarization position angle that we define as

$\psi_{\mathrm{S}}=\frac{1}{2} \arctan \left(-U_{\mathrm{S}}, Q_{\mathrm{S}}\right)$

the minus sign accounts for the conversion of the position angles from the HEALPix (or COSMO) convention to the IAU convention (east of north). We forced the polarization position angles to be in the range $0-180^{\circ}$. The evaluation of the errors on $P_{\mathrm{S}}$ and $\psi_{\mathrm{S}}$ relies on simulations as explained in Appendix A.

For our analysis, we also made use of the full-sky dust extinction map derived in Planck Collaboration XI (2014), which provides discussions and details. This quantity, directly related to the optical depth of the ISM toward the quasars, helps to evaluate the level of contamination of the quasar optical light by the Galactic dust (see Planck Collaboration Int. XXI 2015 and our Sect. 3). For consistency, we also smoothed the $E(B-V)$ map with the same Gaussian kernel FWHM values as for the polarization maps.

\section{Dust contamination scenario: analysis and results}

In the optical, the principal source of polarization contamination from the Galaxy is due to thermal dust (e.g.,
Planck Collaboration Int. XIX 2015; Planck Collaboration Int. XX 2015; Planck Collaboration Int. XXI 2015). If the magnetic field is coherent in a given region of the sky, dust grains align with the lines of the field in that region (Martin 2007 and references therein for viable physical models producing the alignment). The polarization state of an incident light beam passing through this region is then modified. The electric component perpendicular to the magnetic field is anisotropically dimmed by diffusion on the dust grains. An unpolarized incident light beam therefore exits the dusty region with a net polarization parallel to the magnetic field. The diffuse Galactic dust also emits thermally polarized light in the submillimeter spectral band. The polarization of this light is to be preferentially perpendicular to the lines of the magnetic field in which the dust grains are embedded (see, e.g., Martin 2007 for a discussion).

As a consequence, for a given line of sight, polarization vectors in the submillimeter and in the visible are expected to be orthogonal to one another if the optical polarization is only due to dust. If the background quasar has an intrinsic polarization then the dust contamination can be simplistically modeled as the vectorial addition of an ISM polarization (perpendicular to the dust polarized emission) to the intrinsic quasar polarization. An offset toward the perpendicularity can, in principle, be detected statistically. That excess is expected to be better detected the larger the dust-induced optical polarization compared to the quasar intrinsic degree of optical polarization.

Below we introduce the quantities that link dust and quasar polarization data and with which we intend to estimate the eventual contamination of the quasar optical polarization by the intervening Galactic dust.

\subsection{Dust-to-quasar polarization correlation quantities}

As discussed above, optical polarization of a background source that would be only due to depolarization by dust is predicted to be perpendicular to the submillimeter polarization emitted by the dust grains. The relative angle between the measured polarization vectors in the two wavebands can thus be considered as an indicator for dust contamination of the quasar optical polarization data. The relative angle between the two polarization vectors is computed as

$\Delta_{\mathrm{S} / \mathrm{V}}=90-|90-| \psi_{\mathrm{S}}-\psi_{V}||$,

where the angles are expressed in degree. The consecutive absolute values take into account the axial nature of the polarization vectors. The value $\Delta_{\mathrm{S} / \mathrm{V}}$ is defined in the range $0-90^{\circ}$. For a sample of lines of sight, any deviation from uniformity of the $\Delta_{\mathrm{S} / \mathrm{V}}$ distribution toward $90^{\circ}$ could indicate a possible contamination of the sample. Therefore, we consider the $\Delta_{\mathrm{S} / \mathrm{V}}$ as our first dust-to-quasar contamination gauge. The $\Delta_{\mathrm{S} / \mathrm{V}}$ distribution of the 355 comparison measurements is shown in Fig. 1. A clear departure from uniformity is seen. We quantify and discuss this in the next subsection. We note that a departure from uniformity would not provide a sufficient argument to conclude for a contamination. Coincidental position angle correlations need to be taken into account. Such consideration is explored in Sect. 3.5. We also note that the opposite is equally true; a uniform distribution of the $\Delta_{\mathrm{S} / \mathrm{V}}$ is not a sufficient argument to claim for no contamination. For example, large experimental uncertainties can uniformize the angle distribution or the dust-induced polarization could be not sufficient to override completely the intrinsic optical polarization of the background source (see also the discussion in Sect. 4.2.3). As a result, the criterion based on the $\Delta_{\mathrm{S} / \mathrm{V}}$ distribution can only be used to diagnose a 


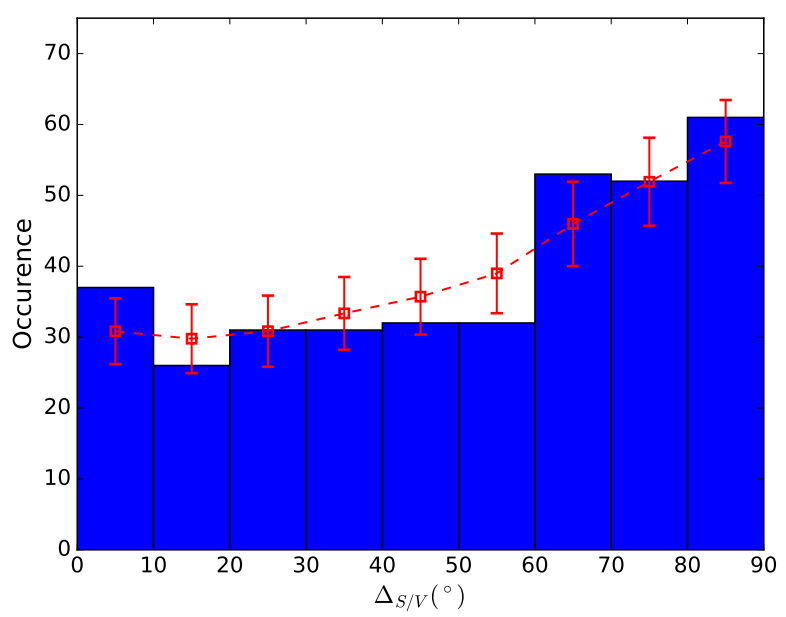

Fig. 1. Histogram of the 355 relative angles $\Delta_{\mathrm{S} / \mathrm{V}}$ computed as in Eq. (2). The observed distribution is significantly not uniform and shows an excess toward $90^{\circ}$, which corroborates a contamination scenario. The central values and error bars given in red account for observational uncertainties. The errors are estimated through $10^{6}$ simulations in which we vary the polarization position angles of the quasars and dust around their values according to the observational uncertainties (see text for details). The central values and error bars correspond to the mean and dispersion in each bin obtained by computing the histogram for each simulation. The average correlation appears weaker because of the blurring of the correlation that the randomization of the angles within their uncertainties produces.

significant contamination of the optical light by the intervening dust.

We complement our study using two polarization ratios discussed in Martin (2007) and that were recently used in Planck Collaboration Int. XXI (2015) to derive ISM physical properties by comparing polarized light from stars in the optical domain ( $V$ band) and thermal dust emission from the same Planck data that we use in this work.

The first polarization ratio we consider is defined as

$R_{\mathrm{P} / \mathrm{p}}=P_{\mathrm{S}} / p_{V}$,

and has units of polarized intensity (here $K_{\mathrm{CMB}}$ ). For Galactic star-related studies, this ratio is used to characterize the efficiency of the dust grains at producing polarized submillimeter emission compared to their ability at polarizing starlight in the visible. In this case, the fundamental difference with starlight based studies is that the quasars of our sample are thought to have an intrinsic degree of linear polarization while the starlight are assumed to be unpolarized. The $R_{\mathrm{P} / \mathrm{p}}$ values are therefore expected to be lower in our study than in Planck Collaboration Int. XXI (2015), for example. Also a consequence of this is that the polarization ratio is defined even when $P_{\mathrm{S}}$ is zero.

Large values of the $R_{\mathrm{P} / \mathrm{p}}$ require at the same time a large polarized dust emission and a low degree of linear polarization of the quasars. The first condition implies dusty regions with a significant alignments of nonspherical dust grains which, in turn, implies an efficient polarizability of incident optical light. The second condition is fulfilled for quasars that are more prone to be affected by dust contamination, i.e., those with the smallest intrinsic degrees of linear polarization.

The other polarization ratio that we consider is defined as

$R_{\mathrm{S} / \mathrm{V}}=\frac{P_{\mathrm{S}} / I_{\mathrm{S}}}{p_{V} / \tau_{V}}$

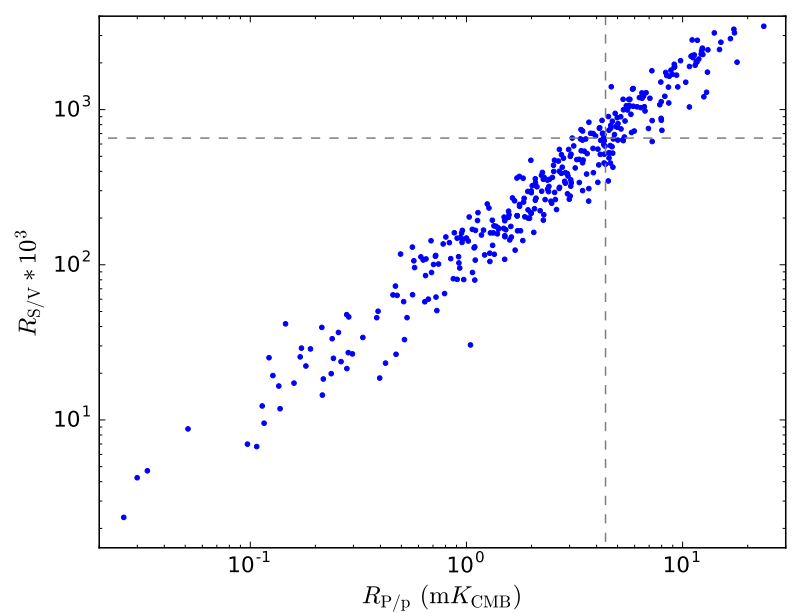

Fig. 2. Scatter plot in the plane $\left(R_{\mathrm{P} / \mathrm{p}}, R_{\mathrm{S} / \mathrm{V}}\right)$ of the 355 measurements of the polarization ratios measured from the Planck maps smoothed with a Gaussian kernel of FWHM of $15^{\prime}$ and the quasar data. Horizontal and vertical dashed lines mark the cuts suggested in Sect. 4.1 to retrieve a $\Delta_{\mathrm{S} / \mathrm{V}}$ distribution that agrees with the hypothesis of uniformity at the $2 \sigma$ level. Our analysis does not allow us to argue for dust contamination for the quasars corresponding to polarization ratio values below these thresholds.

where $\tau_{V}$ is the optical depth to the quasar, measured in the $V$ band. We compute this quantity from the extinction $E(B-V)$ as $\tau_{V}=E(B-V) R_{V} / 1.086$ with $R_{V}$, the ratio of total to selective extinction (e.g., Planck Collaboration Int. XXI 2015). We adopt $R_{V}=3.1$, which is the indicated value for the diffuse ISM (e.g., Fitzpatrick 2004). Our results are independent of this value, which only acts as a scaling factor.

While $R_{\mathrm{P} / \mathrm{p}}$ is defined such that it is sensitive to the polarizing grains alone, the nondimensional ratio $R_{\mathrm{S} / \mathrm{V}}$ somehow weights $R_{\mathrm{P} / \mathrm{p}}$ by the total amount of dust and its extinction effect on the light of the background source. The phenomenology from this ratio is similar to that with $R_{\mathrm{P} / \mathrm{p}}$. The larger the $R_{\mathrm{S} / \mathrm{V}}$ value, the more prone to dust contamination the optical polarized signal. Our aim at considering this quantity is to cross check the results obtained with $R_{\mathrm{P} / \mathrm{p}}$. For clarity, we base our discussion in the core of the paper on $R_{\mathrm{P} / \mathrm{p}}$. The results with $R_{\mathrm{S} / \mathrm{V}}$ are given in the Appendix D.

From a phenomenological point of view and if the optical data set is affected by the Galactic dust at a detectable level, we might expect to observe a correlation between the angle $\Delta_{\mathrm{S} / \mathrm{V}}$ and the polarization ratios. The larger the value of the polarization ratios, the more likely perpendicular the polarization vectors should be. We search for such correlation in Sect. 3.3. Figure 2 shows the scatter plot of the pairs $\left(R_{\mathrm{P} / \mathrm{p}}, R_{\mathrm{S} / \mathrm{V}}\right)$ for the quasar sample.

\subsection{Preliminary results}

We present the distribution of the $355 \Delta_{\mathrm{S} / \mathrm{V}}$ measurements in Fig. 1. The blue histogram corresponds to the $\Delta_{\mathrm{S} / \mathrm{V}}$ that are computed from the position angles of the quasar optical polarization vectors and the $353 \mathrm{GHz}$ dust polarization vectors built from the maps smoothed with a Gaussian kernel of FWHM of $15^{\prime}$. The departure from uniformity is significant and is robust even accounting for observational uncertainties as illustrated in red in Fig. 1 (see Sect. 3.3 for the computation of the uncertainties). A two-sided Kolmogorov-Smirnov test gives a probability that the blue histogram is drawn from a parentuniform distribution of about $2.4 \times 10^{-6}$. This probability is independent of the number of bins. Besides, the cumulativebinomial probability to observe, out of 355212 or more data 
Table 1. Results of uniformity test and historical subsamples.

\begin{tabular}{lccc}
\hline \hline Sample & $N$ & $\left\langle\cos \left(\Delta_{\mathrm{S} / \mathrm{v}}\right)\right\rangle$ & $p_{\text {value }}(\%)$ \\
\hline All & 355 & 0.552 & $<10^{-4}$ \\
$b \geq 30^{\circ}$ & 195 & 0.583 & 0.80 \\
$b \leq-30^{\circ}$ & 160 & 0.514 & $<10^{-4}$ \\
$p_{V} \leq 1.37$ & 178 & 0.537 & $8.0 \times 10^{-4}$ \\
$p_{V}>1.37$ & 177 & 0.567 & 0.15 \\
$b \geq 30^{\circ}, p_{V} \leq 1.32$ & 98 & 0.551 & 0.33 \\
$b \geq 30^{\circ}, p_{V}>1.32$ & 97 & 0.573 & 2.26 \\
$b \leq-30^{\circ}, p_{V} \leq 1.45$ & 80 & 0.482 & $5.0 \times 10^{-4}$ \\
$b \leq-30^{\circ}, p_{V}>1.45$ & 80 & 0.546 & 0.45 \\
$z \leq 1.0$ & 178 & 0.504 & $<10^{-4}$ \\
$z>1.0$ & 177 & 0.600 & 5.78 \\
$b \geq 30^{\circ}, z \leq 0.96$ & 98 & 0.511 & $5.0 \times 10^{-3}$ \\
$b \geq 30^{\circ}, z>0.96$ & 97 & 0.655 & 72.2 \\
$b \leq-30^{\circ}, z \leq 1.02$ & 80 & 0.481 & $5.0 \times 10^{-4}$ \\
$b \leq-30^{\circ}, z>1.02$ & 80 & 0.546 & 0.50 \\
\hline
\end{tabular}

Notes. Probabilities are in percent. The value $N$ is the number of data points in the considered subsamples, $p_{V}$ the degree of linear optical polarization (in \%), and $z$ the redshift of the quasars. The $\left\langle\cos \left(\Delta_{\mathrm{S} / \mathrm{V}}\right)\right\rangle$ is measured from the subsamples and the probabilities $p_{\text {value }}$ are set using $10^{6}$ random simulations of samples of size $N$.

points with $\Delta_{\mathrm{S} / \mathrm{V}}>45^{\circ}$ is found to be $P_{\text {bin }}=1.5 \times 10^{-4}$. This shows that the excess toward the perpendicularity is significant. Alternatively, we consider the mean of the cosines of the relative angles, denoted $\left\langle\cos \left(\Delta_{\mathrm{S} / \mathrm{V}}\right)\right\rangle$. Under the hypothesis that these angles are uniformly distributed in the range $0-90^{\circ}$, the expected value of this mean is $2 / \pi \simeq 0.6366$. Based on Monte Carlo simulations, in which the relative angles are randomly generated according to a flat distribution, we compute the probability to observe by chance a value of $\left\langle\cos \left(\Delta_{\mathrm{S} / \mathrm{V}}\right)\right\rangle$ as extreme as that from the data, the $p$-value of the observation. Out of $10^{6}$ random realizations, none managed to reach the value obtained for the data, leading to a $p$-value of $p_{\text {value }} \lesssim 10^{-6}$. This is more than a $5 \sigma$ deviation from uniformity.

We tested the robustness of our results against experimental errors from both quasar optical polarization data and Galactic thermal dust polarization data. To account for these uncertainties, we generated the polarization position angles, polarized intensity, and degree of linear polarization according to normal distributions centered on the observations and with their corresponding width. The errors for the quasars were taken from the catalog and were propagated from the uncertainties on Stokes obtained by simulations in Appendix A, for the dust. We produced $10^{6}$ of realizations.

From the sample of 355 quasars, several subsamples were considered in the literature (e.g., Hutsemékers 1998; Hutsemékers et al. 2005; Jain et al. 2004; Pelgrims \& Cudell 2014) to identify the regions of the parameter space in which the quasars have their optical polarization vectors more aligned with one another. For completeness and for comparison with these works, we apply such historical cuts and test the uniformity of the corresponding $\Delta_{\mathrm{S} / \mathrm{V}}$ distributions. We thus separate in two approximately equal parts the sample $(i)$ by means of the sky location of the quasars (we separate the northern to the southern Galactic sky), (ii) by means of the degree of linear polarization, and (iii) by means of the redshift $z$ of the quasars. We also consider combinations of these selection criteria. Table 1 summarizes the results using the $\left\langle\cos \left(\Delta_{\mathrm{S} / \mathrm{V}}\right)\right\rangle$ uniformity test. The two other tests lead to very similar results.
Table 2. Results of uniformity test and polarization-ratio subsamples.

\begin{tabular}{lccc}
\hline \hline Sample & $N$ & $\left\langle\cos \left(\Delta_{\mathrm{S} / \mathrm{V}}\right)\right\rangle$ & $p_{\text {value }}(\%)$ \\
\hline$R_{\mathrm{P} / \mathrm{p}} \leq 2.55$ & 178 & 0.610 & 12.4 \\
$R_{\mathrm{P} / \mathrm{p}}>2.55$ & 177 & 0.493 & $<10^{-4}$ \\
$b \geq 30^{\circ}, R_{\mathrm{P} / \mathrm{p}} \leq 2.46$ & 98 & 0.643 & 58.0 \\
$b \geq 30^{\circ}, R_{\mathrm{P} / \mathrm{p}}>2.46$ & 97 & 0.522 & 0.02 \\
$b \leq-30^{\circ}, R_{\mathrm{P} / \mathrm{p}} \leq 2.84$ & 80 & 0.581 & 5.48 \\
$b \leq-30^{\circ}, R_{\mathrm{P} / \mathrm{p}}>2.84$ & 80 & 0.447 & $<10^{-4}$ \\
\hline
\end{tabular}

Notes. Probabilities are in percent, $R_{\mathrm{P} / \mathrm{p}}$ in $\mathrm{mK}_{\mathrm{CMB}}$. The value $N$ is the number of data points in the considered subsample. The $\left\langle\cos \left(\Delta_{\mathrm{S} / \mathrm{V}}\right)\right\rangle$ is measured from the subsample and the probabilities $p_{\text {value }}$ are set using $10^{6}$ random simulations of samples of size $N$.

Under the assumption that the departure from uniformity of the $\Delta_{\mathrm{S} / \mathrm{V}}$ distribution with an excess toward $90^{\circ}$ indicates a contamination of the optical polarization data by Galactic dust, inspection of Table 1 reveals that, the contamination is more noticeable for the part of the quasar sample with a low degree of linear polarization. The contamination of the quasar sample could be significant for the whole southern Galactic sky, even if the departure from uniformity and the predominance for the perpendicularity appears to be stronger for the low-redshift part and/or for the low degree of linear polarization part of the sample. In the northern Galactic cap, however, the contamination seems to involve preferentially the low-redshift part of the sample. The high-redshift part presents even a small deficit, and not an excess, of polarization vectors at more than $45^{\circ}$ to the dust polarization vectors; as opposed to what contamination would produce. It is specifically in that region of the space (angular and redshift coordinates) that the extreme-scale alignments of the quasar optical polarization vectors have been shown to be the more significant (Hutsemékers et al. 2005; Pelgrims \& Cudell 2014) in the northern Galactic sky.

The conclusions from our investigation using dust polarization data agree with those obtained in Hutsemékers et al. (2005), Payez et al. (2010) based on polarized starlight. All their discussions on the implication of the dust contamination on the pertinence of the cosmological-scale alignments of the quasar polarization vectors are thus expected to hold.

In Table 2, we report the same kind of investigation but using the median of the polarization ratio $R_{\mathrm{P} / \mathrm{p}}$ to define the subsamples. Inspection of this table suggests that it is the part of the sample that corresponds to large values of the polarization ratio from which the significant departure from uniformity of the $\Delta_{\mathrm{S} / \mathrm{V}}$ distribution toward $90^{\circ}$ mainly comes from. Indeed, cutting the samples in two equal-size subsamples always leaves a $\Delta_{\mathrm{S} / \mathrm{V}}$ distribution compatible with uniformity for the low values of the polarization ratio. On the contrary large values of $R_{\mathrm{P} / \mathrm{p}}$ correspond to significant deviation from uniformity. This dichotomy is quantified using a two-sample Kolmogorov-Smirnov test. The probability that the $\Delta_{\mathrm{S} / \mathrm{V}}$ distributions of the low and high $R_{\mathrm{P} / \mathrm{p}^{-}}$ value parts are drawn from the same parent distribution is found to be $P_{2 \mathrm{KS}}\left(R_{\mathrm{P} / \mathrm{p}}\right)=0.441 \%$. We investigate further this point in the remainder of the paper.

\subsection{Correlation between $\Delta_{\mathrm{S} / \mathrm{V}}$ and $R_{\mathrm{P} / \mathrm{p}}$}

As discussed in Sect. 3.1, $R_{\mathrm{P} / \mathrm{p}}\left(\right.$ and $\left.R_{\mathrm{S} / \mathrm{V}}\right)$ represents a good parameter according to which the hypothesis of dust contamination can be investigated. In Sect. 3.2 we found that $R_{\mathrm{P} / \mathrm{p}}$ could be correlated to the degree of uniformity of the $\Delta_{\mathrm{S} / \mathrm{V}}$ distribution. 


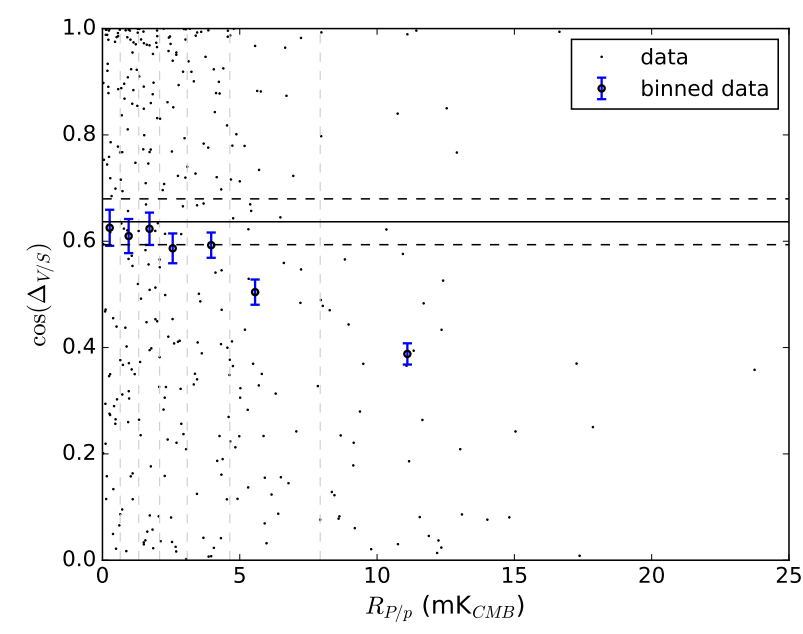

Fig. 3. Scatter plot in the plane $\left(\cos \left(\Delta_{\mathrm{S} / \mathrm{V}}\right), R_{\mathrm{P} / \mathrm{p}}\right)$ and binned data. The error bars correspond to the errors on the mean of $\cos \left(\Delta_{\mathrm{S} / \mathrm{V}}\right)$ in bin that are due to experimental uncertainties on polarization position angles (dust and quasars). Sampling errors in bin are about two times larger. Vertical gray lines show the borders of each bins. They were chosen such that each bin contains 51 data points, except the last one which contains 49 . The horizontal tick line shows the expected mean under the assumption of uniformity in bin and the horizontal dashed lines show the one standard deviation from this mean, computed for 51 data points.

We go further in the investigation of this possible correlation. In Fig. 3 we show the scatter plot of the couple $\left(\cos \left(\Delta_{\mathrm{S} / \mathrm{V}}\right), R_{\mathrm{P} / \mathrm{p}}\right)$. In that figure, we also present binned data (blue points). The adopted sampling is such that each bin contains a fair - and approximately equal - amount of data points and in the meantime, leaves us with as many bins as possible to allow for a detailed study in terms of the $R_{\mathrm{P} / \mathrm{p}}$ values.

As observed in Fig. 3, the larger the $R_{\mathrm{P} / \mathrm{p}}$ value, the smaller the $\left\langle\cos \left(\Delta_{\mathrm{S} / \mathrm{v}}\right)\right\rangle$ value. That is, the larger the $R_{\mathrm{P} / \mathrm{p}}$, the more perpendicular are the optical polarization vectors with respect to the submillimeter vectors. We quantify this correlation using the Spearman's rank-order correlation test. Applied on the pairs $\left(\Delta_{\mathrm{S} / \mathrm{V}}, R_{\mathrm{P} / \mathrm{p}}\right)$, the obtained correlation coefficient is 0.24 and the two-sided probability of obtaining this result by chance is $5.5 \times 10^{-6}$. We further verify this result by means of a permutation test with $10^{6}$ random simulations to evaluate the random distribution of the correlation coefficient. The random realizations were obtained by shuffling the values of the polarization ratio on the $\Delta_{\mathrm{S} / \mathrm{V}}$ values. The resulting distribution of the correlation coefficients, corresponding to the hypothesis of no correlation, is given by the histogram in Fig. 4. This distribution has a mean and a standard deviation of 0.0 and 0.05 , respectively. This leads to the conclusion that the hypothesis of uniformity has to be rejected at 4.5 sigma. The (one-sided) $p$-value of the observed correlation, indicated by the (red) vertical line, is computed as $2.0 \times 10^{-6}$.

The robustness of the correlation regarding the lines of sight with dust data having low $\mathrm{S} / \mathrm{N}$ is discussed in Sect. 4.2. The robustness of our analysis with respect to the value of the FWHM of the Gaussian kernel to smooth the dust polarization maps is discussed in Appendix $\mathrm{B}^{6}$. As shown in Appendix D, this result is also confirmed using the polarization ratio $R_{\mathrm{S} / \mathrm{V}}$ instead of $R_{\mathrm{P} / \mathrm{p}}$.

\footnotetext{
6 We also checked that the deviation from uniformity of the $\Delta_{\mathrm{S} / \mathrm{V}}$ distribution is also observed if we consider the $353 \mathrm{GHz} Q$ and $U$ polarization sky maps given by the COMMANDER component separation algorithm Planck Collaboration X (2016). These maps have a resolution of $10^{\prime}$.
}

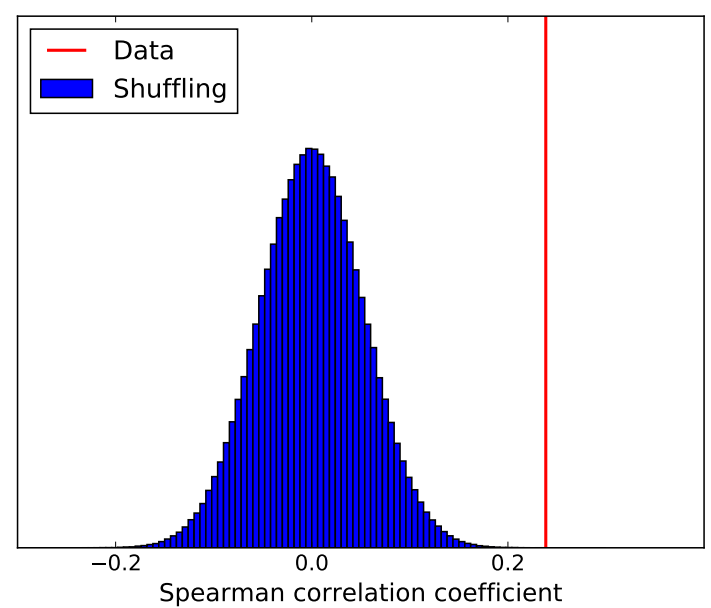

Fig. 4. Spearman's rank-order correlation coefficients between $\Delta_{\mathrm{S} / \mathrm{V}}$ and $R_{\mathrm{P} / \mathrm{p}}$. The histogram corresponds to the distribution obtained by shuffling $10^{6}$ time the $R_{\mathrm{P} / \mathrm{p}}$ on the relative angles $\left(\Delta_{\mathrm{S} / \mathrm{V}}\right)$. The (red) vertical line corresponds to the correlation coefficient obtained from the data.

Table 3. Correlation between $\Delta_{\mathrm{S} / \mathrm{V}}$ and the observables.

\begin{tabular}{lcc}
\hline \hline Observable & $\rho$ & $P_{\text {Spe }}(\%)$ \\
\hline$R_{\mathrm{P} / \mathrm{p}}$ & 0.24 & $5.5 \times 10^{-4}$ \\
$R_{\mathrm{S} / \mathrm{V}}$ & 0.23 & $9.1 \times 10^{-4}$ \\
$\tau_{V}$ & 0.13 & 1.51 \\
$P_{\mathrm{S}}$ & 0.24 & $5.8 \times 10^{-4}$ \\
$p_{\mathrm{S}}$ & 0.21 & $6.6 \times 10^{-3}$ \\
$I_{\mathrm{S}}$ & 0.12 & 1.94 \\
$b$ & -0.08 & 14.6 \\
$l$ & -0.03 & 61.0 \\
$z$ & -0.14 & 0.71 \\
$p_{V}$ & -0.04 & 40.2 \\
\hline
\end{tabular}

Notes. The polarization ratios $R_{\mathrm{P} / \mathrm{p}}$ and $R_{\mathrm{S} / \mathrm{V}}$, the dust-related quantities $\tau_{V}, P_{\mathrm{S}}, p_{\mathrm{S}}, I_{\mathrm{S}}$ and the quasar related quantities $b, l, z, p_{V}$. The two-sided probabilities obtained by the Spearman's rank-order correlation test are reported along with the corresponding correlation coefficient $\rho$.

\subsection{Other correlations involving $\Delta_{S / V}$}

For the sake of completeness, we have searched for similar correlations to those observed between the $\Delta_{\mathrm{S} / \mathrm{V}}$ and $R_{\mathrm{P} / \mathrm{p}}$ but with the various observables, either from the quasar data, the dust data, or a mix of those. These observable are the two polarization ratios $R_{\mathrm{P} / \mathrm{p}}$ and $R_{\mathrm{S} / \mathrm{V}}$, the dust related quantities $\tau_{V}, P_{\mathrm{S}}, p_{\mathrm{S}}$, $I_{\mathrm{S}}$ and the quasar quantities $b, l, z, p_{V}$, all defined above. We led this investigation using the Spearman rank-order correlation test. The results are given in Table 3 . Very significant correlations are detected with the polarization quantities related to Galactic dust.

We also detect a significant correlation between $\Delta_{\mathrm{S} / \mathrm{V}}$ and the redshift of the quasars. This correlation is further discussed in Sect. 4.3.1.

\subsection{Corroborating the contamination scenario}

As mentioned in Sect. 3.2, any deviation from uniformity of the distribution of the $\Delta_{\mathrm{S} / \mathrm{V}}$ angles indicates a possible contamination of the optical polarization sample by foreground Galactic dust. However, a nonuniform $\Delta_{\mathrm{S} / \mathrm{V}}$ distribution is not a selfconsistent proof for a contamination. The opposite being also 
true: uniformity of $\Delta_{\mathrm{S} / \mathrm{V}}$ distribution does not have to be considered as a self-consistent proof of no contamination. Coincidental (un-)correlation of the position angles needs to be investigated. To that concern, the correlation that we have found between $\Delta_{\mathrm{S} / \mathrm{V}}$ and the polarization ratio $R_{\mathrm{P} / \mathrm{p}}$ (and $R_{\mathrm{S} / \mathrm{V}}$ ) is more convincing as arguments in favor of a measurable contamination of the quasar optical polarization sample. Furthermore, the shuffling procedure that we applied in Sect. 3.3 on the pairs $\left(\Delta_{\mathrm{S} / \mathrm{V}}, R_{\mathrm{P} / \mathrm{p}}\right)$ and $\left(\Delta_{\mathrm{S} / \mathrm{V}}, R_{\mathrm{S} / \mathrm{V}}\right)$ proves that the departure from uniformity of the $\Delta_{\mathrm{S} / \mathrm{V}}$ distribution is unlikely accidental but is rather due to contamination by intervening dust.

To make sure that we are not facing a scenario of fortuitous locations of lines of sight, we proceed to the two following additional tests. We rotate the Planck dust polarization maps by longitude steps of five degrees around the north Galactic pole, from $5^{\circ}$ to $355^{\circ}$. For each finite rotation we compute the relative angles between the quasar and dust polarization vectors, the two polarization ratios and finally the Spearman correlation coefficients, as for the data. We further invert the southern and northern hemisphere and proceed to the same analysis. This provides us with a distribution of 142 Spearman's correlation coefficients with which to compare that from the real observations. Out of the 142 realizations by rotation, none show a correlation coefficient of the pairs $\left(\Delta_{\mathrm{S} / \mathrm{V}}, R_{\mathrm{P} / \mathrm{p}}\right)$ as extreme as for the data. The same conclusion holds for the pairs $\left(\Delta_{\mathrm{S} / \mathrm{V}}, R_{\mathrm{S} / \mathrm{V}}\right)$. According to this additional test, the probability that the observed correlations happen by chance is lower than $1 \%$. The Spearman correlation coefficient from the measurements are $0.02 \pm 0.06$ for the two polarization ratios. We applied bootstrap resampling method to verify the reliability of the values of the mean and standard deviation of the distributions. Utilizing $10^{6}$ bootstrap realizations from the distribution of the 142 correlation coefficients we found very consistent values. Given the values of the correlation coefficients for the data, the significance of the observed correlations is about 3.7 sigma. A coincidental nonuniformity of the $\Delta_{\mathrm{S} / \mathrm{V}}$ distribution and of its correlation with polarization ratios are therefore shown as being very unlikely.

We further rely on an additionally shuffling test to strengthen the one-to-one correlation that the quasar polarization data seemingly show with the dust polarization data. We are interested in testing the hypothesis according to which quasar optical polarization and dust submillimeter polarization are correlated one another for each line of sight. We thus shuffle the quasar data $\left(p_{V}\right.$ and $\left.\psi_{V}\right)$ on the dust data $\left(P_{\mathrm{S}}, \psi_{\mathrm{S}},\left(I_{\mathrm{S}}, \tau_{V}\right)\right)$ to build the prior distribution of uncorrelated sample. We proceed to $10^{6}$ reshuffling. The obtained distribution of the null hypothesis (no-correlation) has a mean and a standard deviation of 0.006 and 0.05 , to be compared to 0.0 and 0.05 obtained in Sect. 3.3. As above, fortuitous correlation between dust and quasar polarization data is disfavored given the high significance of the detected correlation $(>4 \sigma)$. We note that according to such a shuffling method, any global systematic (or bias) or any global offset in the data set should be taken into account in the computation of the distribution of the null hypothesis.

The analyses and results presented in this subsection point toward the identification of detectable contamination of the quasar optical polarization data by the intervening Galactic dust. We made sure that the observed contamination signatures are unlikely to arise by chance. We are confident in our claim given the significance of the reported departure from uniformity of $\Delta_{\mathrm{S} / \mathrm{V}}$ distribution, the correlation between these angles with the polarization ratios, and the correlation between the polarization of the quasar and dust.

\section{Discussion: mitigating the dust contamination}

In this section, we exploit the correlation between the Galactic thermal dust data and the quasar optical polarization in order to explore the actual impact of dust contamination on the reported extreme-scale alignments of quasar optical polarization vectors without reproducing the cosmological analysis.

\subsection{Search for a new quality criterion}

Figure 3 shows that the departure from uniformity of the $\Delta_{\mathrm{S} / \mathrm{V}}$ distribution is likely due to the lines of sight having the largest values of the polarization ratio $R_{\mathrm{P} / \mathrm{p}}$. It is therefore appealing to introduce a quality criterion of the quasar optical polarization based on the values of this ratio. We provide the first investigation in that direction. We show below that this parameter can be used to remove the data points that are significantly affected by the dust and therefore obtain a sample of quasar optical polarization measurements for which the dust contamination is not significant. Let us emphasize that this does not prevent an actual contamination by the dust. It rather suggests that the contamination is not strong enough to be clearly detected. Therefore, in this case, the orientation of the quasar optical polarization is expected to be not too affected such that we can trust it relates to the intrinsic polarization.

In Fig. 5 (top), starting with the original sample of $355 \Delta_{\mathrm{S} / \mathrm{V}}$ measurements, we gradually reduce the sample size by removing those data points having largest value of the polarization ratio $R_{\mathrm{P} / \mathrm{p}}$. It is shown that gradually removing the part of the sample corresponding to the higher values of the $R_{\mathrm{P} / \mathrm{p}}$ ratio leads to $\left\langle\cos \left(\Delta_{\mathrm{S} / \mathrm{V}}\right)\right\rangle$ values more in agreement with the hypothesis of a uniform distribution. This is illustrated in Fig. 5 (bottom) where we show the one-sided $p$-value of the observation for each removed sampling fraction. These are the probabilities that a sample drawn from a parent uniform distribution leads to a value larger than that of the observed $\left\langle\cos \left(\Delta_{\mathrm{S} / \mathrm{V}}\right)\right\rangle$ of the truncated sample. The one-sided p-values of the sample corresponding to truncation of 20,30 , and $40 \%$ are respectively, $1.2 \%, 4.6 \%$, and $15.2 \%$. Removing about 30 percent of the sample is sufficient to retrieve a $\cos \left(\Delta_{\mathrm{S} / \mathrm{V}}\right)$ distribution for which the hypothesis of uniformity cannot be rejected at the two sigma level.

We further show, in Fig. 6, that the polarization ratio is a good parameter at discriminating quasar optical polarization data that are strongly contaminated by dust. To that aim, we compare the $\left.\left\langle\cos \left(\Delta_{\mathrm{S} / \mathrm{V}}\right)\right)\right\rangle$ obtained after removing the $30 \%$ corresponding to the largest $R_{\mathrm{P} / \mathrm{p}}$ values from the sample to that when randomly removing $30 \%$ of the sample. It is shown that $(i)$ the $\left\langle\cos \left(\Delta_{\mathrm{S} / \mathrm{V}}\right)\right\rangle$ values obtained by random truncation of the sample keep deviating significantly from the hypothesis of uniformity, (ii) the values of $\left\langle\cos \left(\Delta_{\mathrm{S} / \mathrm{V}}\right)\right\rangle$ corresponding to the sample truncated by means of $R_{\mathrm{P} / \mathrm{p}}$ do not correspond to a random truncation of the sample, and finally (iii) that the latter values do not violate the hypothesis of a uniform distribution of the $\Delta_{\mathrm{S} / \mathrm{V}}$ angles at the two sigma level. In Appendix D, we show that similar result is obtained for $R_{\mathrm{S} / \mathrm{V}}$ and also for the other values of the FWHM of the Gaussian kernel used to smooth the dust maps.

In order to minimize the impact from dust contamination in the quasar sample, it appears relevant to discriminate the data points with respect to their value of the polarization ratios. Our analysis suggests removing at least all data points with either $R_{\mathrm{P} / \mathrm{p}} \geq 4.4 \mathrm{mK}_{\mathrm{CMB}}$ or $R_{\mathrm{S} / \mathrm{V}} \geq 6.5 \times 10^{-3}$ from the original sample. However these values depend on the width of the smoothing kernel used to compute the polarization ratios from the polarized maps. These threshold values have to be regarded with caution. 

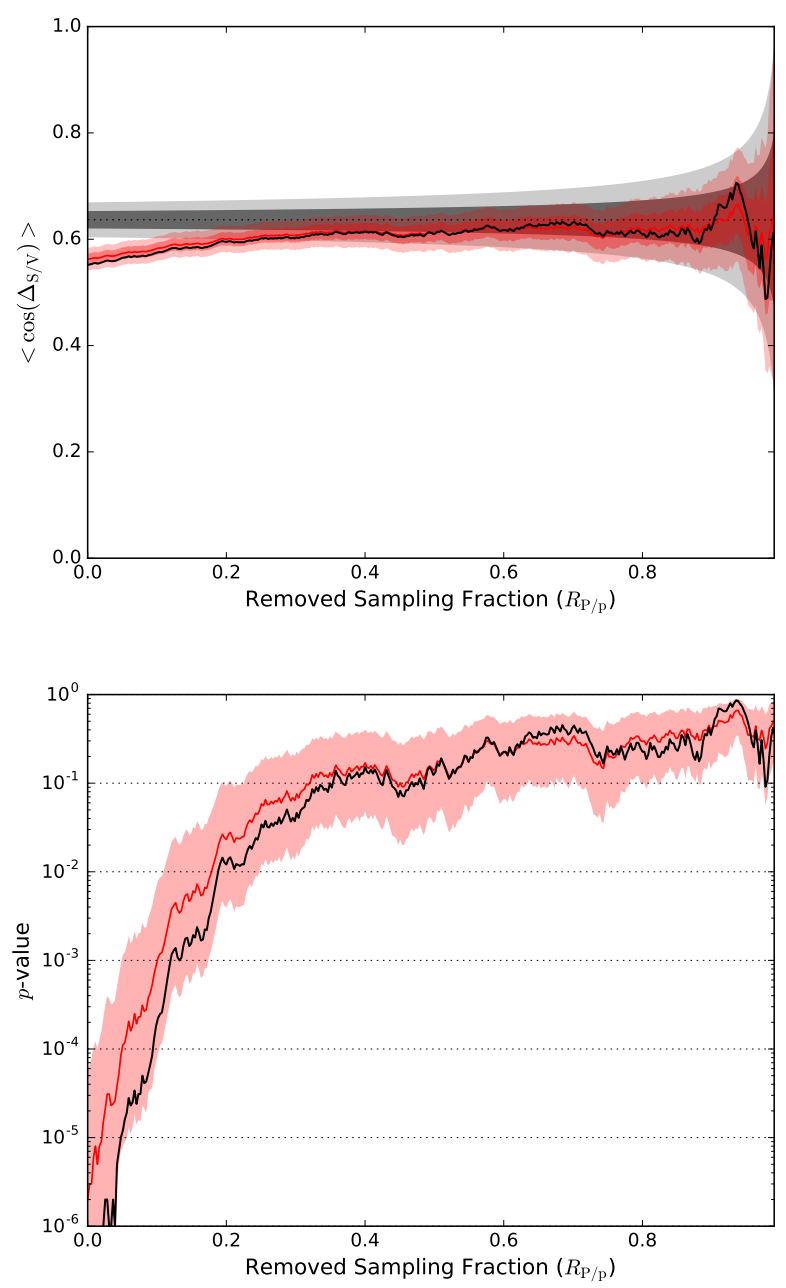

Fig. 5. Top panel, in black: $\left\langle\cos \left(\Delta_{\mathrm{S} / \mathrm{v}}\right\rangle\right.$ value from the data as a function of the fraction of the sample that is removed according to the $R_{\mathrm{P} / \mathrm{p}}$ values of the data points (see text). The red line and shaded regions indicate the mean and the one and two sigma contours from the observational errors on the polarization position angles (dust and quasar). The gray shaded regions correspond to the one and two sigma deviations around the expected value (black dot horizontal line), assuming a uniform distribution of $\Delta_{\mathrm{S} / \mathrm{V}}$ angles. Bottom panel: under the hypothesis of uniform distribution of $\Delta_{\mathrm{S} / \mathrm{V}}$, the black line is the $p$-value of the observation as a function of the removed fraction of the sample. The red line and shaded region correspond to the p-values computed for the mean and the one standard deviation from the mean of the observation accounting for the position angles uncertainties.

First because they are derived from the sample itself and second, because the remaining fraction of the sample contains the lines of sight for which the dust polarization quantities are poorly known owing to the Planck sensitivity and the very diffuse character of the ISM (see below).

\subsection{Caveats and mitigation}

A question arises about how and how much our results are sensitive to the part of the sample that corresponds to the lines of sight for which only low $\mathrm{S} / \mathrm{N}$ data are available for the Galactic thermal dust polarized emission. We address this question below in investigating the impact of the polarization bias on the significance of the reported correlation. We further infer the impact of the low $\mathrm{S} / \mathrm{N}$ data points on the suggested quality criteria to be imposed to the quasar sample. We finally provide physical

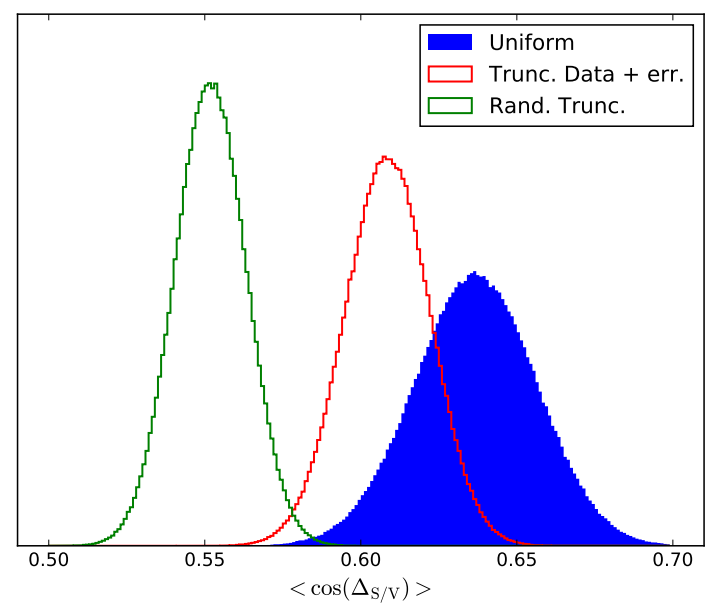

Fig. 6. Filled blue histogram: distribution of $\left\langle\cos \left(\Delta_{\mathrm{S} / \mathrm{V}}\right)\right\rangle$ for $10^{6}$ realizations of uniform $\Delta_{\mathrm{S} / \mathrm{V}}$ distributions for a sample size of $0.7 \times 355$. The red unfilled histogram corresponds to the values obtained for the data when removing the $30 \%$ of the sample with the higher values of the $R_{\mathrm{P} / \mathrm{p}}$ ratio. This distribution takes into account the errors on the polarization position angles. The green unfilled histogram shows $10^{6}$ realizations of $30 \%$ random truncation of the original sample.

arguments to not discard those data points with low $\mathrm{S} / \mathrm{N}$ dust polarization data.

\subsubsection{Robustness of the correlation and low $\mathrm{S} / \mathrm{N}$ data}

By construction, noisy polarization data are subject to a positive bias that artificially produces larger values of the polarized intensity (e.g., Wardle \& Kronberg 1974). While the quasar optical polarization data have been corrected for this bias in the catalog, we did not attempt to correct the dust polarization data for it.

First, let us note that $\sigma_{\mathrm{P}_{\mathrm{S}}}$ is largely uniform at high Galactic latitudes owing to the scan strategy of Planck. As a consequence, in our sample, the $\mathrm{S} / \mathrm{N}$ depends quasi-linearly on the $P_{\mathrm{S}}$ values, which implies that the low $\mathrm{S} / \mathrm{N}$ of the polarized intensities correspond to low $P_{\mathrm{S}}$ values which, in turn, are more prone to the polarization bias. As a result, within the sample from which we reported a correlation between $R_{\mathrm{P} / \mathrm{p}}$ and $\Delta_{\mathrm{S} / \mathrm{V}}$ (see Sect. 3.3), it is the part with the low $R_{\mathrm{P} / \mathrm{p}}$ values that is the more likely affected by the polarization bias. Because the polarization bias leads to artificially increased values of $P_{\mathrm{S}}$ and thus of $R_{\mathrm{P} / \mathrm{p}}$ values, the bias can only act to reduce the significance of the correlation. Indeed, in principle the polarization bias affects only points to the left of Fig. 3 (low $P_{\mathrm{S}}$ values), by shifting these points to the right. The correction of the bias would therefore in principle lead to a small shift of these points to the left. Correcting for polarization bias would therefore result in a tighter correlation.

Therefore, keeping the data points with low $\mathrm{S} / \mathrm{N}$ mitigates the detection of the dust contamination, which is already significant. We thus found unnecessary to correct for this effect using, for example, debiasing methods such as that presented by Plaszczynski et al. (2014). Meanwhile, we had repeated most of the analyses but removed the sight lines for which $P_{\mathrm{S}}<\sigma_{\mathrm{P}_{\mathrm{S}}}$. With that reduced sample we found consistent results but with lower significance. For instance, the probability given by the Spearman correlation test that the correlation between $\Delta_{\mathrm{S} / \mathrm{V}}$ and $R_{\mathrm{P} / \mathrm{p}}$ is due to chance was found to be $1.0 \times 10^{-4}$ instead of $5.5 \times 10^{-6}$. This is the opposite of what we expect from the above. This is because we are dominated by the sampling variance rather than by the observational uncertainties. 


\subsubsection{Quality criterion and low $\mathrm{S} / \mathrm{N}$ data}

While investigating the fraction of the sample that has to be removed to define a quasar optical polarization sample where the possible dust contamination is minimized, we have to worry about the role of the sight lines with low $\mathrm{S} / \mathrm{N}$ dust data. It is clear that those data points likely contribute to the uniformity of the $\Delta_{\mathrm{S} / \mathrm{V}}$ distribution. This is because large uncertainties on the dust polarization position angles would act to smear the correlation between dust and quasars, if there is any. We can decide to reject those lines of sight from our analysis. If we reject all lines of sight with $P_{\mathrm{S}}<\sigma_{\mathrm{P}_{\mathrm{S}}}$ and reproduce the truncation analysis presented in Sect. 4.1, we find that the $\Delta_{\mathrm{S} / \mathrm{V}}$ distributions keep deviating from uniformity, with a $p$-value as high as 1$5 \%$, even for large values of the removed fraction. This does not implies that a fraction of the corresponding quasar sample cannot be used for cosmological inference. Indeed, for low $R_{\mathrm{P} / \mathrm{p}}$ values, irrespective of the noise level of the dust polarization data, the contamination by dust of the quasar optical polarization data might be too inefficient to smear the intrinsic quasar optical polarization.

As a corollary, the above discussion shows that we need the sight lines for which the dust data are dominated by the noise to retrieve a distribution of the $\Delta_{\mathrm{S} / \mathrm{V}}$ that is compatible with uniformity, i.e., that the contamination is only marginally detected based on the relative angle criterion. It is thus legitimate to attempt to figure out if this uniformity is physical or due to lacking quality of the dust polarization data. This point is addressed below.

\subsubsection{Low $\mathrm{S} / \mathrm{N}$ and inefficient contamination}

As already mentioned, low $\mathrm{S} / \mathrm{N}$ dust polarization data corresponds to low $P_{\mathrm{S}}$ in our sample. Based on a phenomenological model of the polarized dust emission (see, e.g., Pelgrims et al. 2018; Pelgrims \& Macías-Pérez 2018, for a review and similar discussion), we know that low $P_{\mathrm{S}}$ values correspond either to lines of sight with low dust column density or to lines of sight along which depolarization is at play because of conspiring geometry of the Galactic magnetic field. Indeed, the Galactic magnetic field could be such that the observed polarized intensity is low because of the integration of many different preferred directions along the line of sight. In the former case low total intensity of the dust is also to be expected while in the latter case the dust intensity are expected to be large as resulting from the integration of the dust density along the line of sight. We note that in case of depolarization, even if no particular correlation would be observed between the orientations of the submillimeter and optical polarization vectors, we could not safely consider the measured quasar optical polarization as being intrinsic to the sources. In such a case our analysis would become senseless.

To verify that we are not facing such a scenario, we consider those lines of sight for which the $\mathrm{S} / \mathrm{N}$ of the dust polarized signal is low. We observed that they correspond preferentially to low $I_{\mathrm{S}}$ values, corresponding to low column density. This has been tested by means of a two-sample Kolmogorov-Smirnov test that leads to a probability below $3.0 \times 10^{-4}$ that the $I_{\mathrm{S}}$ distribution of the subsample is a random subset from that of the whole sample. This situation is even more pronounced by means of the optical depth $\tau_{V}$. This points toward a scenario according to which there is not enough dust along the sight lines to significantly modify the polarization state of the optical light from the background quasars. Therefore, the observed uniformity of the $\Delta_{\mathrm{S} / \mathrm{V}}$ distribution of this subsample of low $\mathrm{S} / \mathrm{N}$ dust data is seemingly physical. Relying on this argument, we shall prefer to not reject that part of the sample from our analysis.

Even if the above does not allow us to thoroughly conclude for no-dust contamination of the subsample of the quasar optical polarization corresponding to the sight lines with low $\mathrm{S} / \mathrm{N}$, we have to acknowledge the arguments pointing toward a low contribution of the Galactic dust on those polarization data.

\subsection{Do the extreme-scale alignments survive?}

The result obtained in the previous subsections show that, based on our contamination criterion, Galactic ISM contamination is not detected at the two sigma level for about 70 percent of the quasar optical polarization sample presented by Hutsemékers et al. (2005). We argue that this fraction of the sample could be used for cosmological investigations as we expect the impact of the dust contamination on the cosmological analysis to be small. It is beyond the scope of the present paper to reproduce the study of the large-scale anisotropies of the quasar optical polarization vector orientations. Nevertheless let us present some hints that points toward the fact that the extreme-scale alignments cannot fully be explained by a dust contamination scenario.

\subsubsection{Redshift dependence}

In Sect. 3.4 we reported on a significant correlation between $\Delta_{\mathrm{S} / \mathrm{V}}$ and the redshift of the quasars. This actually reflects the redshift dependence of the preferred orientation of the quasar optical polarization vectors (at least in the northern Galactic hemisphere). Indeed, in the northern hemisphere, quasars seem to have - on average - their polarization vectors more randomly oriented with respect to the dust (second line of Table 1). Interestingly, low-redshift quasars have their polarization vector preferentially perpendicular to the dust polarization vectors. The tendency is at the opposite for high-redshift quasars. This dichotomy is well observed by comparing the 12 th and the 13th lines of Table 1.

In the past, Hutsemékers (1998), Hutsemékers \& Lamy (2001) and Hutsemékers et al. (2005) reached compatible conclusions considering starlight polarization. This observational fact provided an actual strong argument against the hypothesis that dust contamination would be at the origin of the reported extreme-scale alignments of the quasar optical polarization vectors. The reason is that the correction of a strong Galactic contamination at low redshift would automatically imply much stronger alignments at high redshift as the lines of sight of the two regions pass through the same Galactic space (Payez et al. 2010). Therefore, in at least one of the two gigaparsec-scale regions of the space defined by Hustemékers et al. toward the north Galactic pole, the quasars are expected to have their optical polarization vectors effectively aligned one another. This argument holds as far as the lines of sight to those quasars indeed probe the same ISM. This was not an a priori guarantee given the, sometimes rapid, spatial variation (on sky) of the column density of the polarizing dust and the induced polarization direction.

In this work, by highlighting a redshift dependence of the preferred orientation of the quasar optical polarization vectors directly compared to the dust polarization vectors, we provide further credits to the old arguments, discarding a scenario of conspiring dust features. 

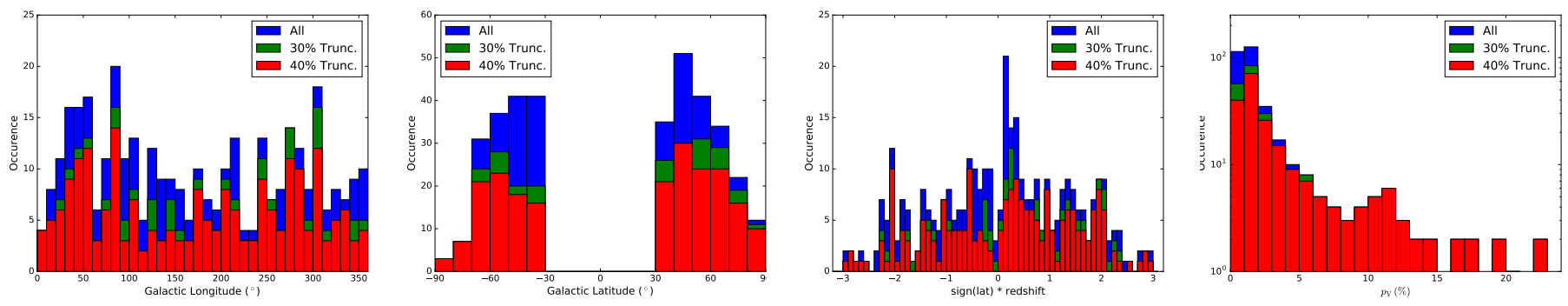

Fig. 7. Distribution of the parameters attached to the quasars without and with selections by means of the $R_{\mathrm{P} / \mathrm{p}}$ ratio. From left to right: Galactic longitude, Galactic latitude, signed resdhift (to differentiate the northern $(>0)$ and southern Galactic cap $(<0)$ ) and degree of linear polarization. The filled blue histogram represents the full catalog, green and red show the 30 and $40 \%$ truncation, respectively. We note the log-scale for the last plot.

\subsubsection{Alignment regions and strong contamination}

From previous works (Hutsemékers 1998; Hutsemékers et al. 2005; Pelgrims \& Cudell 2014), we know that the alignments of the polarization vectors are stronger, i.e., more significant, in well-defined regions of the three-dimensional space. We have checked that the quality criteria based on polarization ratios does not favor a depopulation of those regions, compared to other places. For completeness, we give in Fig. 7 the histograms of the physical parameters of the quasars belonging to the whole catalog and with 30 and 40 percent truncation according to the $R_{\mathrm{P} / \mathrm{p}}$ values. It can be seen that the selection occurs preferentially in some regions of the parameter space ${ }^{7}$. However, these are not those in which the Gpc-scale alignments were reported as being the more significant. For example, the so-called A1 region was defined according to the limits (see, e.g., Hutsemékers et al. 2005): $168^{\circ} \leq \mathrm{RA} \leq 218^{\circ}$, Dec $\leq 50^{\circ}$ and $1.0 \leq z \leq 2.3$, where RA and Dec stand for the right ascension and declination of the quasars. In the whole sample, this region contains 56 quasars. Among those, 46 (38) pass the selection of the 30 (40) percent truncation based on $R_{\mathrm{P} / \mathrm{p}}$ values, to be compared to the expected 39.1 (33.6).

Based on the above, we cannot argue for significant changes in the relevance of the extreme-scale alignments of the quasar optical polarization vectors because the alignments were discovered in a sample having half of its current size and we do not find stronger contamination by intervening Galactic dust of the quasars belonging to the regions where the alignments are known to be the more significant. To the question of whether or not the extreme-scale alignments of the quasar optical polarization vectors survive to the Galactic dust contamination scenario, the answer is likely yes. A clear demonstration of this claim would require further investigation relying on a large number of simulations and on the elaboration of a framework within which the contamination by intervening Galactic dust would be reliably modeled and removed from the quasar data.

\section{Conclusions and perspectives}

Hutsemékers (1998), Hutsemékers \& Lamy (2001) and Hutsemékers et al. (2005) have reported alignments of quasar optical linear polarization vectors extending over cosmologicalscale regions of the Universe. Severe quality criteria, which considerably limit the sample size, were adopted to reduce at best the Galactic contamination of the quasar optical polarization data by dust. The authors concluded that even if the resulting

\footnotetext{
7 We note that this was already known from studies relying on the starlight optical polarization inquiries (Hutsemékers et al. 2005).
}

sample is unavoidably contaminated to some level by the ISM, this contamination would never be able to account fully for the observed characteristics of the extreme-scale alignments. The redshift dependence of the alignment directions is one of the characteristics of the alignments that cannot be easily explained with Galactic foregrounds (see Payez et al. 2010 and also our Sect. 4.3.1).

In this paper, we reinvestigate the degree of contamination of these optical polarization data by Galactic dust using Planck data instead of starlight optical polarization measurements. Our goal was to make sure that the contamination level of the quasar optical polarization sample is low and to investigate the possibility that complex Galactic dust features could account for the alignments. We led our investigation using the Planck full-sky maps at $353 \mathrm{GHz}$, which capture the diffuse thermal dust polarized emission. This observable is complementary and independent from starlight-based polarization estimation of the ISM. It has the advantage to have an homogeneous sky sampling and to be representative of the whole integration of the dust along the line of sight.

First, our analysis reveals a significant deviation $(>5 \sigma)$ from uniformity of the distribution of the relative angles between the polarization vectors of the quasars and the dust $\left(\Delta_{\mathrm{S} / \mathrm{V}}\right)$. There is a significant tendency toward the perpendicularity of those vectors. Second, we found significant correlations $(\sim 4 \sigma)$ between the relatives angles and two polarization ratios that are computed for each line of sight to a quasar. The larger the values of the polarization ratios $\left(R_{\mathrm{P} / \mathrm{p}}\right.$ or $\left.R_{\mathrm{S} / \mathrm{V}}\right)$, the more perpendicular the optical polarization vectors to the dust polarization vectors. Therefore, using dust maps, we were able to diagnose a stronger contamination of the quasar optical polarization data by the Galactic dust than was previously thought (Hutsemékers 1998; Hutsemékers \& Lamy 2001; Hutsemékers et al. 2005). Indeed, relying on criteria based on the distribution of the relative angles and on two polarization ratios, we revealed a measurable effect of the intervening Galactic dust on the quasar optical polarization data. We further showed that, based on the relative angle criterion, the contamination is only marginally detected when removing at least roughly 30 percent of the sight lines with high values of the polarization ratios. This suggests that for the remaining lines of sight the contamination by the intervening Galactic dust is likely inefficient at smearing the intrinsic optical polarization of the quasars. This reduced sample could then be used for cosmological inference. We thus provide a new quality criterion based on the polarization ratio values to minimize at best the ISM contamination.

While a definitive proof requires further developments and analyses, we found arguments that reinforce previous claims according to which the ISM cannot be responsible for the 
cosmological-scale alignments of the quasar optical polarization vectors. The cosmological relevance of the extreme-scale alignments of the quasar polarization vectors is thus expected to be unchanged as also discussed at the end of Sect. 4.3. We further reinforced the argument against a Galactic contamination for the quasars that have high redshift and that are located in the northern Galactic cap. The tendency of the $\Delta_{\mathrm{S} / \mathrm{V}}$ distribution is indeed opposed to what a contamination would produce.

Further inquiries would require reproducing the analyses presented in Hutsemékers et al. (2005) and Pelgrims \& Cudell (2014) with the restricted sample, i.e., applying the polarizationratio selection. Alternatively, providing a fair modeling of Galactic dust contamination of the optical polarization data, a decontamination of the sample should be investigated. The observational characteristics of the extreme-scale alignments of the quasar polarization vectors shall be adapted accordingly. While this is well beyond the purpose of this work, this task is mandatory in a search for the physical scenario responsible for these striking observations. For such an inquiry, additional quasar optical polarization data will be required. Quasars showing a low degree of linear polarization should particularly be included in the sample as they could provide upper limits to the dust contribution of the optical polarized signal. Together with polarization data of Galactic stars, they could be used to fit the parameters of the contamination model. Unfortunately, and to the best of our knowledge, there is yet no publicly available large quasar optical polarization sample of that sort. Hopefully this work would motivate the elaboration of such a sample.

Large-scale correlations of the quasar polarization vectors have also been observed at radio wavelengths $(8.4 \mathrm{GHz}$; Pelgrims \& Hutsemékers 2015) in a large sample of flatspectrum radio sources compiled by Jackson et al. (2007). While there were arguments against the hypothesis of strong Galactic contamination, it should be worthwhile to perform a comparison similar to that presented in this work, but between the synchrotron full-sky map released by Planck and the point-like radio source polarization vectors. To this regard, the polarization maps of the diffuse emission and the point-like source catalog at low frequencies $(11-20 \mathrm{GHz})$ that will be released by the RADIOFOREGROUNDS ${ }^{8}$ project using data from the QUIJOTE experiment (Rubiño-Martín et al. 2017) will constitute a considerable advantage to lead such inquiries in the northern equatorial hemisphere.

Acknowledgements. I thank Céline Combet, Damien Hutsemékers, Juan Macías-Pérez, Florian Ruppin, and Nicolas Ponthieu for their generous advice and the inspiring and guiding discussions that led to a significant improvement of this manuscript. I also thank Vincent Guillet for a late discussion and for pointing out a unit mistake to me. I acknowledge my referee R. Genova-Santos for his help in clarifying the discussion and in highlighting the conclusions of this work. This work has been partially funded by the European Union's Horizon 2020 research and innovation program under grant agreement number 687312. We acknowledge the use of data from the Planck/ESA mission, downloaded from the Planck Legacy Archive, and of the Legacy Archive for Microwave Background Data
Analysis (LAMBDA). Support for LAMBDA is provided by the NASA Office of Space Science. Some of the results in this paper have been derived using the HEALPix (Górski et al. 2005) package.

\section{References}

Agarwal, N., Kamal, A., \& Jain, P. 2011, Phys. Rev. D, 83, 065014 Axon, D. J., \& Ellis, R. S. 1976, MNRAS, 177, 499

Burstein, D., \& Heiles, C. 1982, AJ, 87, 1165

Chang, Z., Wang, S., \& Li, X. 2012, Euro. Phys. J. C, 72, 1838

Ciarcelluti, P. 2012, Mod. Phys. Lett. A, 27, 1250221

Das, S., Jain, P., Ralston, J. P., \& Saha, R. 2005, J. Cosmol. Astropart. Phys., 6, 002

di Serego Alighieri, S. 2015, Int. J. Mod. Phys. D, 24, 1530016

Fitzpatrick, E. L. 2004, in Astrophys. Dust, eds. A. N. Witt, G. C. Clayton, \& B. T. Draine, ASP Conf. Ser., 309, 33

Górski, K. M., Hivon, E., Banday, A. J., et al. 2005, ApJ, 622, 759

Heiles, C. 2000, AJ, 119, 923

Hutsemékers, D. 1998, A\&A, 332, 410

Hutsemékers, D., \& Lamy, H. 2001, A\&A, 367, 381

Hutsemékers, D., Cabanac, R., Lamy, H., \& Sluse, D. 2005, A\&A, 441, 915

Hutsemékers, D., Borguet, B., Sluse, D., Cabanac, R., \& Lamy, H. 2010, A\&A, 520, L7

Hutsemékers, D., Payez, A., Cabanac, R., et al. 2011, in Astronomical Polarimetry 2008: Science from Small to Large Telescopes, eds. P. Bastien, N. Manset, D. P. Clemens, \& N. St-Louis, ASP Conf. Ser., 449, 441

Hutsemékers, D., Braibant, L., Pelgrims, V., \& Sluse, D. 2014, A\&A, 572, A18 Jackson, N., Battye, R. A., Browne, I. W. A., et al. 2007, MNRAS, 376, 371 Jain, P., Narain, G., \& Sarala, S. 2004, MNRAS, 347, 394

Joshi, S. A., Battye, R. A., Browne, I. W. A., et al. 2007, MNRAS, 380, 162 Kuvschinova, E. V., \& Panov, V. F. 2014, Russ. Phys. J., 57, 418

Martin, P. G. 2007, in EAS Pub. Ser., eds. M. A. Miville-Deschênes, \& F. Boulanger, 23, 165

Mathewson, D. S., \& Ford, V. L. 1970, MNRAS, 74, 139

Payez, A., Cudell, J. R., \& Hutsemékers, D. 2008, in AIP Conf. Ser., eds. J. Cugon, J. P. Lansberg, J. Cugon, \& N. Matagne, 1038, 211

Payez, A., Hutsemékers, D., \& Cudell, J. R. 2010, in AIP Conf. Ser., eds. D. B. Tanner, \& K. A. van Bibber, 1274, 144

Pelgrims, V. 2016, PhD thesis [arXiv:1604.05141]

Pelgrims, V., \& Cudell, J. R. 2014, MNRAS, 442, 1239

Pelgrims, V., \& Hutsemékers, D. 2015, MNRAS, 450, 4161

Pelgrims, V., \& Hutsemékers, D. 2016, A\&A, 590, A53

Pelgrims, V., \& Macías-Pérez, J. F. 2018, A\&A, submitted [arxiv:1807. 10516] Pelgrims, V., Macías-Pérez, J. F., \& Ruppin, F. 2018, A\&A, submitted [arxiv:1807.10515]

Planck Collaboration VI. 2014, A\&A, 571, A6

Planck Collaboration XI. 2014, A\&A, 571, A11

Planck Collaboration XXX. 2014, A\&A, 571, A30

Planck Collaboration X. 2016, A\&A, 594, A10

Planck Collaboration XII. 2018, A\&A, submitted [arxiv:1807.06212]

Planck Collaboration Int. XIX. 2015, A\&A, 576, A104

Planck Collaboration Int. XX. 2015, A\&A, 576, A105

Planck Collaboration Int. XXI. 2015, A\&A, 576, A106

Planck Collaboration Int. XXII. 2015, A\&A, 576, A107

Plaszczynski, S., Montier, L., Levrier, F., \& Tristram, M. 2014, MNRAS, 439, 4048

Poltis, R., \& Stojkovic, D. 2010, Phys. Rev. Lett., 105, 161301

Rubiño-Martín, J. A., Génova-Santos, R., Rebolo, R., et al. 2017, in Highlights on Spanish Astrophysics IX, eds. S. Arribas, A. Alonso-Herrero, F. Figueras, et al., 99

Sluse, D., Hutsemékers, D., Lamy, H., Cabanac, R., \& Quintana, H. 2005, A\&A, 433, 757

Tiwari, P., \& Jain, P. 2015, MNRAS, 447, 2658

Urban, F. R., \& Zhitnitsky, A. R. 2010, Phys. Rev. D, 82, 043524

Wardle, J. F. C., \& Kronberg, P. P. 1974, ApJ, 194, 249

8 http://www.radioforegrounds.eu 


\section{Appendix A: Dust polarized data: smoothing and error estimation}

We use the DR2 Planck HFI $353 \mathrm{GHz}$ Stokes polarization maps $\left(I_{\mathrm{S}}, Q_{\mathrm{S}}\right.$ and $U_{\mathrm{S}}$ ) obtained from the full mission with the five fullsky surveys. We smooth the original maps in order to increase the $\mathrm{S} / \mathrm{N}$ or, accordingly, to reduce the variance in pixel. The price paid is a loss in resolution of the maps altogether with an increase in beam difference between the diffuse dust emission and the point-like quasar. We shall thus find the smallest smoothing length that leads to reliable dust polarization quantities. We use the smoothing function from the Python HEALPix package (Górski et al. 2005). This function uses a Gaussian kernel for the smoothing of input maps and allows us to handle easily polarization quantities. We investigated four values of the FWHM of the Gaussian kernel: $5^{\prime}, 10^{\prime}, 15^{\prime}$ and $20^{\prime}$. Owing to the intrinsic map resolution, this corresponds to an effective resolutions of about $7.03^{\prime}, 11.15^{\prime}, 15.79^{\prime}$, and $20.60^{\prime}$, respectively. From these maps we can compute smoothed polarization quantities.

We evaluate the uncertainties in the smoothed maps using a Monte Carlo approach via the maps of the block-diagonal perpixel covariance matrices released by Planck at $N_{\text {side }}=2048$. We adopt the gross approximation that the errors on Stokes parameters are independent of a Gaussian ${ }^{9}$. We proceed as follows: (i) For each pixel, and for each Stokes parameter $\hat{\mathcal{S}}^{i}$, we generate a random realization according to a normal distribution centered on the data with a standard deviation taken as the square root of the corresponding diagonal element from the covariance matrix given in the Planck data, $\sqrt{C_{\mathcal{S S}}}$. (ii) We proceed to the smoothing of the polarization maps, keeping the $N_{\text {side }}$ parameter to 2048. (iii) We extract the Stokes parameters in pixels that contain the quasars. (iv) We run from (i) to (iii) 1000 times. This allows us to evaluate the mean and the standard deviation for the smoothed $I_{\mathrm{S}}, Q_{\mathrm{S}}$ and $U_{\mathrm{S}}$. As for the errors on the smoothed quantities, we attribute the values of the corresponding standard deviations obtained through the simulations. Let us note that these errors are likely overestimated since the noise of the data is accounted twice while generating the simulations. The noise is indeed already included in the measurements from which we generate the random realizations and there is no easy way to remove the noise with confidence. As a result, the significance of detected correlations quoted in the paper are lower bounds and, therefore, can be seen as conservative.

From the smoothed $I_{\mathrm{S}}, Q_{\mathrm{S}}$, and $U_{\mathrm{S}}$ and their estimated errors, we compute the smoothed polarized intensities $P_{\mathrm{S}}$, the polarization position angles $\psi_{\mathrm{S}}$, and their (overestimated) uncertainties $\left(\sigma_{\mathrm{P}_{\mathrm{S}}}, \sigma_{\psi_{\mathrm{S}}}\right)$.

For the 355 lines of sight toward the quasars, the fractions that pass the selection $P_{\mathrm{S}} \geq \sigma_{\mathrm{P}_{\mathrm{S}}}$ are $70.1 \%, 71.5 \%, 80.0 \%$, and $86.5 \%$ for the FWHM smoothing kernel of $5^{\prime}, 10^{\prime}, 15^{\prime}$, and $20^{\prime}$ respectively. As a comparison, only about 60 percent of these lines of sight fulfill the above quality criterion if we consider the original maps. We choose the FWHM value of $15^{\prime}$ to conduct our discussion in the core of the paper as it provides us with a fair compromise between polarized data quality and relative beam difference between the dust and the quasars. We do not reject from our analysis the 20 percent of the data that do not fulfill the above criterion. This is because we might be interested in finding those quasars for which the optical polarization

\footnotetext{
9 We do not expect a significant effect of this approximation on our results. The principal argument is that it turned out that we are dominated and limited by the sampling variance rather than observational uncertainties.
}

is not too affected by the intervening Galactic dust. The sight lines corresponding to poorly detected dust polarized intensity especially represent good candidate regions where dust has negligible effects on polarization inquiries. Therefore, we prefer to keep these regions in our analyses. Nevertheless, we investigate the impact of the inclusion of those lines of sight in the paper and in particular in Sect. 4.2. Additionally, and as demonstrated in the next sections, we conducted our analysis in parallel for the other FWHM values and found consistent results. Furthermore, in the core of the paper, we took into account the observational uncertainties while deriving the most important results.

\section{Appendix B: Uniformity of $\Delta_{\mathrm{S} / \mathrm{V}}$ and FWHM values}

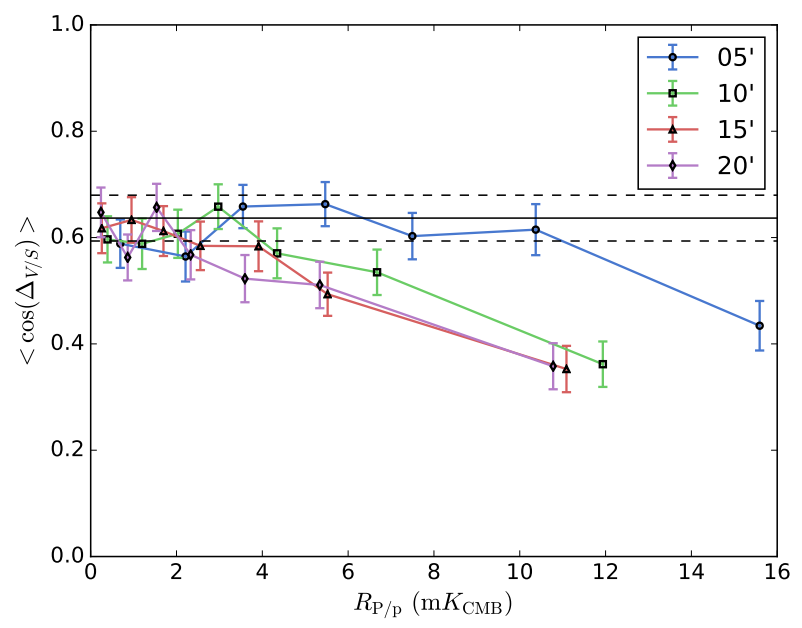

Fig. B.1. Same as for Fig. 3 but for different values of the smoothing of the maps. Error bars represent the sampling uncertainties in bins that we computed as the mean standard errors, i.e., the dispersion in bin divided by the square root of the number of data points in bin. The significant departure from uniformity in the last bin is observed for all tested FWHM values.

The probabilities given by a one-sample Kolmogorov-Smirnov test that the distributions of $\Delta_{\mathrm{S} / \mathrm{V}}$ obtained for the FWHM of $5^{\prime}$, $10^{\prime}, 15^{\prime}$, and $20^{\prime}$ are uniform are found to be $3.95,0.04,2.4 \times 10^{-4}$ and $2.6 \times 10^{-4}$ percent. This illustrates the fact that the departure from uniformity of the distribution of the relative angles between the quasar optical polarization vectors and the dust polarization vectors is robust against the choice of the smoothing value. We checked that this is also the conclusion reached by means of the other tests used in Sects. 3.2 and 3.3. The reason why the departure is less significant for small values of the FWHM is likely because the uncertainties on the dust polarization angles smear the actual correlation. Also, the fact that the probabilities in Table B.1 decrease with increasing FWHM is consistent with the view that the magnetic field has a high degree of coherence at high Galactic latitudes.

In Fig. B.1, we reproduce the results obtained in Fig. 3 for the four FWHM values. The trend that we reported in Sect. 3.3 for $F W H M=15^{\prime}$ is co

nfirmed. The larger the values of the polarization ratios, the more likely perpendicular the quasar optical polarization vectors from the dust polarization vectors. It is worth mentioning that the reason why the polarization ratios tend to smaller values when the FWHM increases comes from beam depolarization. The polarization intensity $P_{\mathrm{S}}$ can indeed get smaller while averaging the fluctuating $Q_{S}$ and $U_{S}$ Stokes parameters in the beam. The Spearman's rank-order correlation coefficients and the 
Table B.1. Spearman rank-order correlation test between $\Delta_{\mathrm{S} / \mathrm{V}}$ and the polarization ratios $R_{\mathrm{P} / \mathrm{p}}$ and $R_{\mathrm{S} / \mathrm{v}}$ for the different FWHM values.

\begin{tabular}{lccccc}
\hline \hline FWHM & \multicolumn{2}{c}{$R_{\mathrm{P} / \mathrm{p}}$} & & \multicolumn{2}{c}{$R_{\mathrm{S} / \mathrm{V}}$} \\
\cline { 2 - 3 } \cline { 5 - 6 } & $\rho$ & $P_{\text {Spe }}(\%)$ & & $\rho$ & $P_{\text {Spe }}(\%)$ \\
\hline $5^{\prime}$ & 0.08 & 12.44 & & 0.11 & 4.40 \\
$10^{\prime}$ & 0.19 & 0.39 & & 0.19 & $3.5 \times 10^{-2}$ \\
$15^{\prime}$ & 0.24 & $5.5 \times 10^{-4}$ & & 0.23 & $9.1 \times 10^{-4}$ \\
$20^{\prime}$ & 0.25 & $5.5 \times 10^{-4}$ & & 0.25 & $2.2 \times 10^{-4}$ \\
\hline
\end{tabular}

Notes. The values $\rho$ and $P_{\text {Spe }}$ are, respectively, the correlation coefficient computed for the pairs and the two-sided probabilities that such correlation is due to chance.

probabilities that the correlations happen by chance are reported in Table B.1 for the four FWHM values and two polarization ratios $R_{\mathrm{P} / \mathrm{p}}$ and $R_{\mathrm{S} / \mathrm{V}}$.

\section{Appendix C: Uniformization of $\Delta_{\mathrm{S} / \mathrm{V}}$ distribution}

An interesting result obtained in the paper is that the entire quasar optical polarization sample does not appear to be significantly affected by the Galactic dust. Removing about $30 \%$ of the sample corresponding to the largest values of the polarization ratios leads to only marginal deviation of the $\Delta_{\mathrm{S} / \mathrm{V}}$ distribution. This has been set using the smoothing value of $15^{\prime}$. Similar results are reached with the other FWHM values. This is illustrated in Fig. C.1 where we reproduce Fig. 5 (top) for the four tested FWHM values but without considering observational uncertainties. Taking into account observational uncertainties (not shown on the figures), the fraction of the original sample that needs to be truncated to obtain a $\Delta_{\mathrm{S} / \mathrm{V}}$ distribution that agrees with uniformity at the $2 \sigma$ level is between about 20 and 40 percent for all the studied FWHM values. That is, 80 to 60 percent of the quasar optical polarization sample appear sufficiently decoupled from the Galactic dust polarization such that we can trust the optical polarization to be intrinsic to the quasars and not too contaminated by intervening Galactic dust.

\section{Appendix D: Polarization ratio $\boldsymbol{R}_{\mathrm{S} / \mathrm{V}}$}

In Sect. 3.1, we discussed two polarization ratios $R_{\mathrm{P} / \mathrm{p}}$ and $R_{\mathrm{S} / \mathrm{V}}$ that mix observable quantities from the optical and submillimeter. As defined, $R_{\mathrm{P} / \mathrm{p}}$ is only sensitive to the polarizing dust grains. The $R_{\mathrm{S} / \mathrm{V}}$ ratio, however, also encodes the effect of nonaligned grains on optical background source light.

For clarity, we presented in the core of the paper only the results of the analyses obtained with the $R_{\mathrm{P} / \mathrm{p}}$ ratio. As we performed in parallel all the analyses considering $R_{\mathrm{S} / \mathrm{V}}$, we show the main results in Figs. D.1-D.4. These correspond to Figs. 3, B.1, 5, and C.1, respectively. As illustrated by these figures, we obtained very consistent results considering either $R_{\mathrm{S} / \mathrm{V}}$ or $R_{\mathrm{P} / \mathrm{p}}$. This could have been expected in light of the observed correlation between the two polarization ratios in Fig. 2.

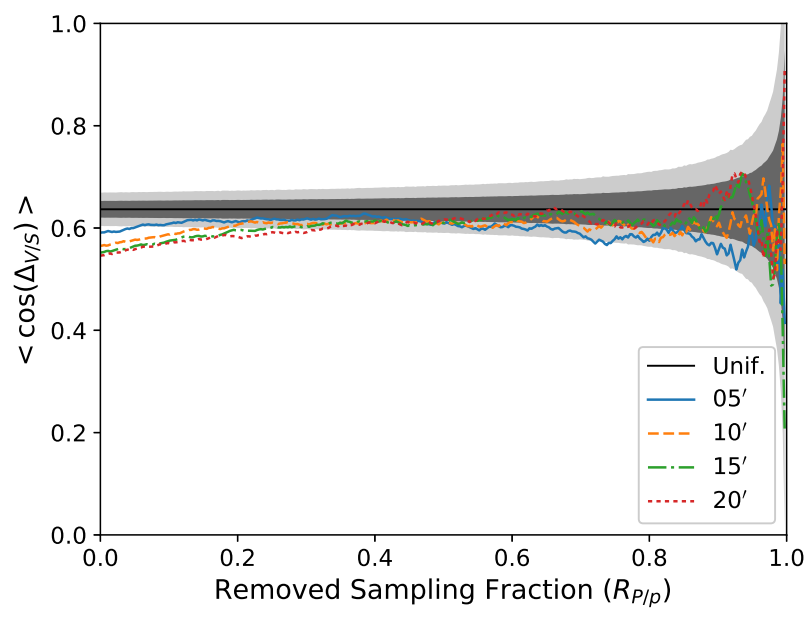

Fig. C.1. Gray shaded regions: one and two sigma deviations around the expected value (black horizontal line), assuming a uniform distribution of $\Delta_{\mathrm{S} / \mathrm{V}}$ angles. The different lines refer to the different values of the FWHM used to evaluate the $R_{\mathrm{P} / \mathrm{p}}$ polarization ratio. They represent the $\left\langle\cos \left(\Delta_{\mathrm{S} / \mathrm{V}}\right\rangle\right.$ values from the data as a function of the fraction of the sample that is gradually removed while decreasing the $R_{\mathrm{P} / \mathrm{p}}$ selection threshold.

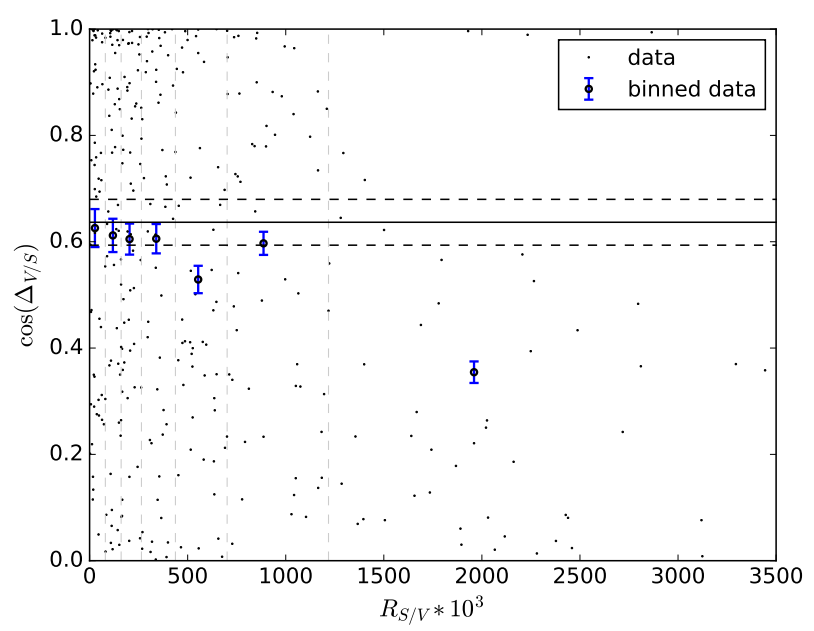

Fig. D.1. Same as for Fig. 3 but for the pairs $\left(\cos \left(\Delta_{\mathrm{S} / \mathrm{V}}\right), R_{\mathrm{S} / \mathrm{V}}\right)$.

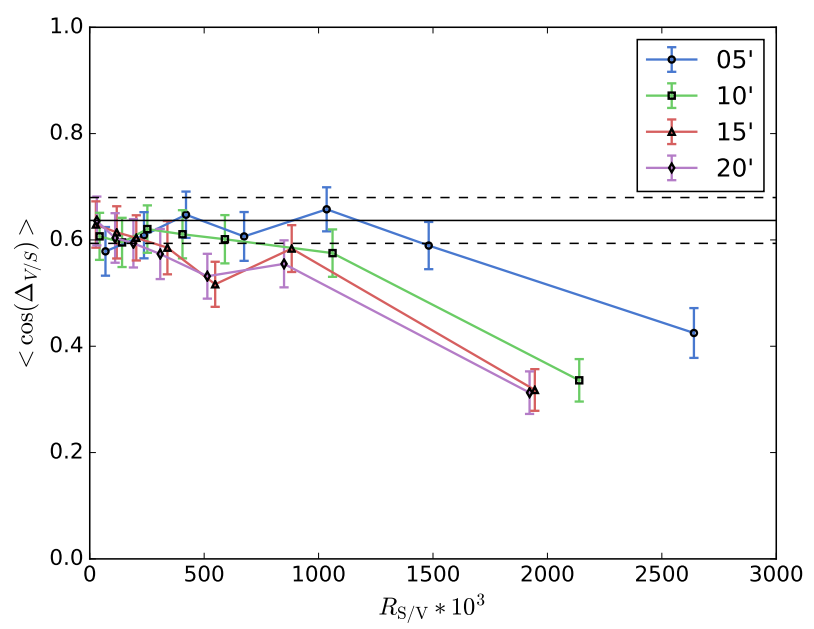

Fig. D.2. Same as for Fig. B.1 but for the pairs $\left(\cos \left(\Delta_{\mathrm{S} / \mathrm{V}}\right), R_{\mathrm{S} / \mathrm{V}}\right)$. 
A\&A 622, A145 (2019)

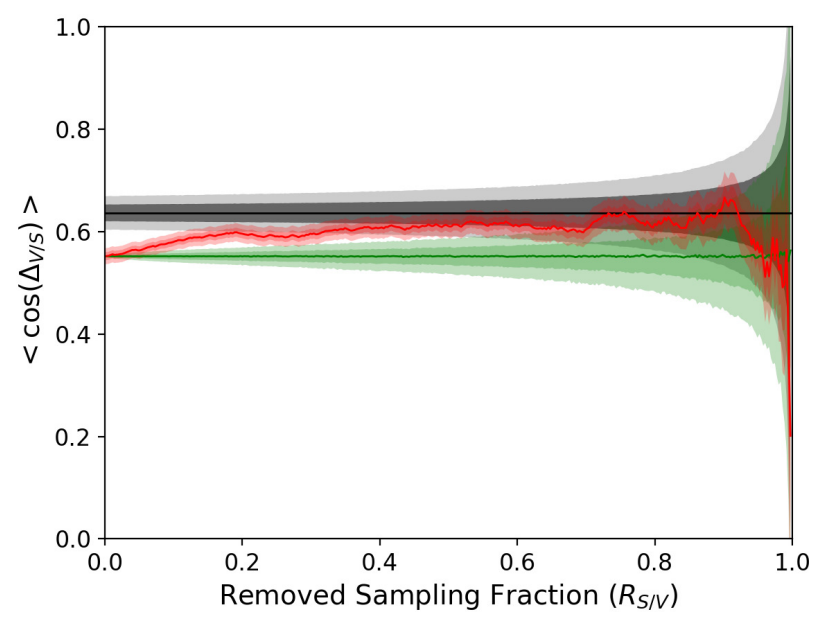

Fig. D.3. Same as for Figs. 5 and 6 but with the polarization ratio $R_{\mathrm{S} / \mathrm{v}}$. The mean and the $1 \sigma$ and $2 \sigma$ regions of the distribution of $\left\langle\cos \left(\Delta_{\mathrm{S} / \mathrm{v}}\right\rangle\right.$ obtained with random truncation is illustrated in green.

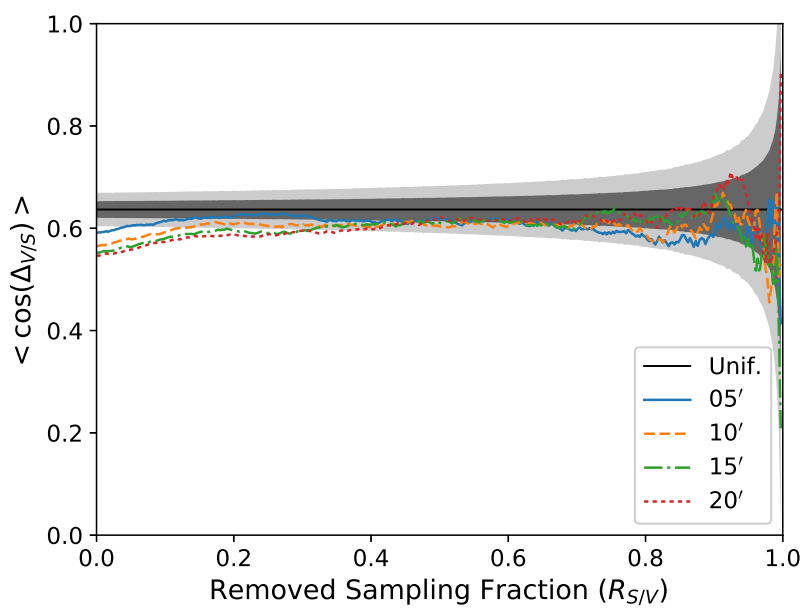

Fig. D.4. Same as for Fig. C.1 but for the $R_{\mathrm{S} / \mathrm{v}}$ polarization ratio. 